\title{
A description of sources detected by INTEGRAL during the first 4 years of observations ${ }^{\star}$
}

\author{
A. Bodaghee ${ }^{1,2}$, T. J.-L. Courvoisier ${ }^{1,2}$, J. Rodriguez ${ }^{3}$, V. Beckmann ${ }^{4}$, N. Produit ${ }^{1,2}$, D. Hannikainen ${ }^{5}$, E. Kuulkers ${ }^{6}$, \\ D. R. Willis ${ }^{1}$, and G. Wendt ${ }^{1}$
}

1 INTEGRAL Science Data Centre, Chemin d'Ecogia 16, 1290 Versoix, Switzerland

e-mail: arash . bodaghee@obs . unige.ch

2 Observatoire Astronomique de l'Université de Genève, Chemin des Maillettes 51, 1290 Sauverny, Switzerland

CEA-Saclay/DSM/DAPNIA/SAp, 91191 Gif-sur-Yvette, France

4 NASA Goddard Space Flight Center, Astrophysics Science Division, Greenbelt, MD 20771, USA

5 Observatory, PO Box 14, 00014 University of Helsinki, Finland

${ }^{6}$ ISOC, ESA/ESAC, Urb. Villafranca del Castillo, PO Box 50727, 28080 Madrid, Spain

Received 12 January 2007 / Accepted 23 February 2007

\section{ABSTRACT}

\begin{abstract}
Context. In its first 4 years of observing the sky above $20 \mathrm{keV}$, INTEGRAL-ISGRI has detected 500 sources, around half of which are new or unknown at these energies. Follow-up observations at other wavelengths revealed that some of these sources feature unusually large column densities, long pulsations, and other interesting characteristics.

Aims. We investigate where new and previously-known sources detected by ISGRI fit in the parameter space of high-energy objects, and we use the parameters to test correlations expected from theoretical predictions. For example, the influence of the local absorbing matter on periodic modulations is studied for Galactic High-Mass X-ray Binaries (HMXBs) with OB supergiant and Be companions. We examine the spatial distribution of different types of sources in the Milky Way using various projections of the Galactic plane, in order to highlight signatures of stellar evolution and to speculate on the origin of the group of sources whose classifications are still uncertain.

Methods. Parameters that are available in the literature, such as positions, photoelectric absorption $\left(N_{\mathrm{H}}\right)$, spin and orbital periods, and distances or redshifts, were collected for all sources detected by ISGRI. These values and their references are provided online.

Results. ISGRI has detected similar numbers of X-ray Binaries and Active Galactic Nuclei (AGN). The former group contains new members of the class of HMXBs with supergiant stellar companions. Usually, this type of object presents strong intrinsic absorption which leads to a peak emission in an energy range that ISGRI is ideally suited to detect. Thanks to these additional systems, we are able to show that HMXBs are generally segregated in plots of intrinsic $N_{\mathrm{H}}$ versus the orbital period of the system and versus the spin period of the pulsar, based on whether the companion is a Be or an OB supergiant star. We also find a tentative but expected anticorrelation between $N_{\mathrm{H}}$ and the orbital period, and a possible and unexpected correlation between the $N_{\mathrm{H}}$ and the spin period. While only a handful of new Low-Mass X-ray Binaries (LMXBs) have been discovered, there are many sources that remain unclassified and they appear to follow a spatial distribution typical of Galactic sources (especially LMXBs) rather than extragalactic sources.
\end{abstract}

Key words. gamma rays: observations - catalogs - X-rays: binaries - Galaxy: stellar content

\section{Introduction}

In just over 4 years, INTEGRAL-IBIS/ISGRI (Lebrun et al. 2003; Ubertini et al. 2003) has detected $\sim 300$ previously-known sources in the hard $\mathrm{X}$ to soft $\gamma$-ray band $(20-100 \mathrm{keV})$, and discovered $\sim 200$ sources that were previously unknown at these energies. We will hereafter refer to the latter sources as IGRs ${ }^{1}$ (for INTEGRAL Gamma-Ray sources). Generally, these sources were detected by creating long-exposure mosaic images captured by ISGRI (e.g., Bird et al. 2007). The INTEGRAL core programme (Winkler et al. 2003) is beginning to fill in underexposed regions of the sky.

Most of the sources that ISGRI has detected are Low and High-Mass X-ray Binaries (LMXBs and HMXBs, respectively), or Active Galactic Nuclei (AGN). Both LMXBs and

\footnotetext{
* Table 1 and references are only available in electronic form at http://www. aanda.org

1 An updated list of IGRs can be found at http://isdc . unige. ch/ rodrigue/html/igrsources.html
}

HMXBs feature a compact object such as a neutron star (NS) or a black hole (BH) accreting material from a companion star: a faint old dwarf in LMXBs $\left(M \lessgtr 1 M_{\odot}\right)$, a bright young giant in HMXBs $\left(M \gtrsim 10 M_{\odot}\right)$, or sometimes an intermediate-mass companion. Accretion typically occurs via Roche-lobe overflow in LMXBs or through the wind in HMXBs. An accretion disk can be found in both types of systems and is an important component of the optical/UV and X-ray emission from AGN and LMXBs.

Subclasses exist within the 3 most common groups. In the case of HMXBs, the spectral type of the stellar companion determines the sub-classification beyond the NS or BH nature of the compact object. A majority of HMXBs host main-sequence (MS) Be stars that have not filled their Roche lobe (Waters \& van Kerkwijk 1989). These systems are usually transient with flares produced whenever the sometimes wide and eccentric orbit brings the compact object close to its companion. Persistent HMXBs are typically accompanied by an evolved supergiant (SG) O or B star whose wind steadily feeds the compact 
object. Their variability stems from inhomegeneities in the wind. Similarly, LMXBs can be classified based on the type of compact object (NS or BH) it has. Neutron star LMXBs can be divided further into $\mathrm{Z}$ or Atoll sources depending on the tracks they follow in a color-color diagram. The 2 primary groups of AGN are Seyfert 1 and 2, with the latter being more absorbed and showing narrow emission lines only.

Our understanding of the different populations of INTEGRAL sources is limited by the large number of sources about which very little is known. Roughly half of all IGRs remain unclassified. The nature of these sources is difficult to elucidate given that many are faint or transient. Furthermore, the images, spectrum, and timing analysis gathered from a single energy range are usually insufficient to classify an object. Information from other wavelengths such as soft X-rays, infrared or radio are necessary to help identify the nature of a source. For example, radio emission can be the signature of a jet or pulsar, while the optical spectral type can help distinguish between LMXBs and HMXBs, and the redshift can place it at extragalactic distances. Follow-up observations with soft X-ray telescopes (i.e. Chandra, RXTE, Suzaku, Swift and $X M M$-Newton) can provide fine timing analyses which enable short-period modulations to be found, and they can describe the shape of the continuum below ISGRI's $\sim 20 \mathrm{keV}$ lower limit, in an energy range where potential photoelectric absorption $\left(N_{\mathrm{H}}\right)$ and iron fluorescence lines are detectable. Precise $\mathrm{X}$-ray coordinates from Chandra, Swift or XMM-Newton can be used to search for counterparts in dedicated radio, optical, and IR observations or in catalogues. However, many sources are clustered in the Galactic center and along the plane, which, because of the density of stars and the amount of obscuring dust, can hinder the identification of the optical/IR counterpart.

Perhaps the most interesting result from follow-up observations is that a number of IGRs present column densities that are much higher than would be expected along the line of sight. These large absorptions are therefore intrinsic and could be the reason these sources eluded discovery with previous (softer) $\mathrm{X}$-ray missions. The first new source discovered by INTEGRAL is IGR J16318-4848 (Courvoisier et al. 2003) which is one of the most absorbed Galactic sources known with $N_{\mathrm{H}} \sim 2 \times$ $10^{24} \mathrm{~cm}^{-2}$ or roughly 2 orders of magnitude more than the intervening Galactic material (Dickey \& Lockman 1990). Since this discovery, other sources joined the growing class of heavilyobscured X-ray sources described by Walter et al. (2004) and Kuulkers (2005).

A certain number of these absorbed sources consist of X-ray pulsars: e.g. IGR J16320-4751 (Rodriguez et al. 2006), IGR J16393-4643 (Bodaghee et al. 2006), and IGR J17252-3616 (Zurita Heras et al. 2006). Their persistent emission, their long pulse periods ( $\sim 1 \mathrm{ks})$, and their large column densities suggest that these systems are likely to be SG HMXBs, with the NS deeply embedded in the wind of its massive stellar companion (Walter et al. 2006). These kinds of systems are still a minority compared to Be HMXBs, but INTEGRAL is expanding their ranks.

Supergiant Fast X-ray Transients (SFXT: see Sguera et al. (2005) for a review) are another type of object whose numbers are increasing thanks to INTEGRAL. These objects are HMXBs whose X-ray emission is characterised by short strong outbursts (a peak flux of up to a Crab or more during a few seconds to a few hundred seconds), sometimes with recurrence timescales that can reach several hundred days. Despite the intensity of their outbursts, SFXTs are usually not detected in deep mosaic images because the accumulation of exposure time attenuates their significance. Therefore, the search for SFXTs involves scanning archival light curve data and short-exposure mosaic images for rapid bursts from known transients or at new locations.

ISGRI has also detected other types of Galactic objects such as Cataclysmic Variables (CVs), Supernova Remnants (SNRs), Pulsar Wind Nebulae (PWN), Anomalous X-ray Pulsars (AXPs), etc., which are referred to henceforth as Miscellaneous.

The most extensive catalogues of sources detected by ISGRI are the catalogues of Bird et al. (2006) and Bird et al. (2007). These catalogues represent fairly large and homogeneous samples that can be used to study the general characteristics of populations of high-energy sources (e.g. Lebrun et al. 2004; Dean et al. 2005; Lutovinov et al. 2005; Beckmann et al. 2006b).

This research presents the parameters of all sources detected by ISGRI and reported between its launch on Oct. 17, 2002, until Dec. 1, 2006. Absorption values, pulse and orbital periods, and distances or redshifts were collected from the literature and were used to study the populations of high-energy sources, to test various correlations expected from theoretical predictions, and to investigate where the new and previously-known sources detected by ISGRI fit in the parameter space of high-energy objects.

\section{Data and analysis}

We selected all sources from Version 27 of the INTEGRAL General Reference Catalogue (Ebisawa et al. 2003) which were detected by ISGRI (i.e. those with "ISGRI_FLAG==1"). These flags were set to the value of 1 as soon as confirmation of a detection by ISGRI is announced in an article, conference proceeding, Astronomer's Telegram or IAU circular. Therefore, the completeness of the sample is difficult to evaluate given that by definition, the present sample contains all sources that were detected above $20 \mathrm{keV}$ while within the ISGRI FOV at some point during the last $\sim 4$ years, without considering the detection significance, nor the amount of exposure time that was required to make the detection.

The exposure map that can be seen in Fig. 1 was created by accumulating all public pointings in revolutions 30-484 (UTC: $11 / 1 / 2003-1 / 10 / 2006)$. Due to the core programme observation strategy, the Galactic centre (GC) is heavily exposed $\left(t_{\exp }>10 \mathrm{Ms}\right)$ whereas some regions have less than $10 \mathrm{ks}$ of exposure time dedicated to them. The exposure is uneven along the Galactic plane as well, with exposure biases in the directions of the spiral arms. The sensitivity limit of a source in the most exposed regions is as low as $\sim 1 \mathrm{mCrab}$ for a transient object detected at the $6 \sigma$ level (Bird et al. 2006). In fact, some sources were detected only because the instrument serendipitously caught a flaring event. Using the fact that the $\log (N)-\log (S)$ relation for extragalatic sources follows a power law with a slope of $-3 / 2$ (Forman et al. 1978), we can estimate a sensitivity limit of $\$ 5 \mathrm{mCrab}\left(=3.78 \times 10^{-11} \mathrm{erg} \mathrm{cm}^{-2} \mathrm{~s}^{-1}\right.$ in $\left.20-40 \mathrm{keV}\right)$ for our sample based on where the distribution of AGN from Bird et al. (2006) deviates from the expected slope.

A number of IGRs have soft X-ray counterparts that were sometimes detected by earlier missions. For example, IGR J16393-4643 was known as AX J1639.0-4642 by ASCA, and IGR J17252-3616 as EXO 1722-360 by EXOSAT, and many IGRs have ROSAT counterparts (Stephen et al. 2006). Since ISGRI was the first to detect them above $20 \mathrm{keV}$, it is legitimate to group them together as a population of new soft $\gamma$-ray sources. They can then be compared to sources detected by ISGRI that were previously known to emit above $20 \mathrm{keV}$ (e.g., Crab, Vela X-1, etc.). Note that the so-called previously-known 


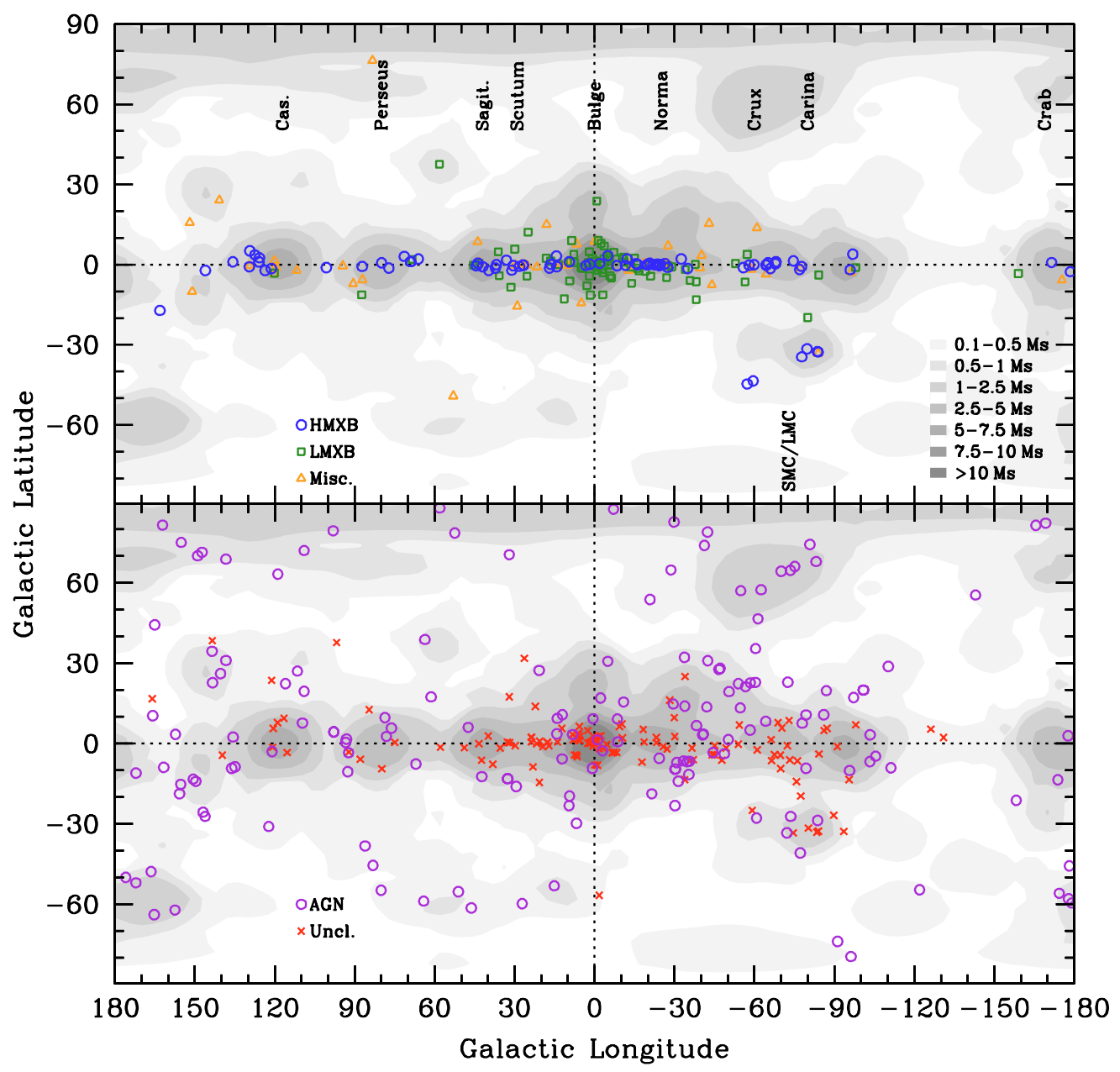

Fig. 1. Spatial distribution in Galactic coordinates of sources detected so far by ISGRI. The figure at the top presents the distributions of HMXBs (stars), LMXBs (squares) and miscellaneous sources (triangles). The figure at the bottom displays extragalactic sources (circles) and unclassified sources (crosses). The directions to the spiral arm tangents and other areas of interest are indicated, as are the cumulative exposure times at each location (from public data in revs. 30-484). The number of sources in each class is listed in Table 2.

sources actually include a few objects that were discovered after the launch of INTEGRAL (e.g. by HETE, RXTE or Swift).

The name or position of each source in our sample was queried to the SIMBAD and ADS servers for references that could provide any of the following parameters: position and error radius, classification, column density $\left(N_{\mathrm{H}}\right)$, spin period, orbital period, and distance (or redshift). Besides a rough X-ray position, very little is known about some sources, while other sources were so thoroughly studied that choices had to be made between sometimes conflicting values (notably $N_{\mathrm{H}}$ and distance). The index of parameters that we have constructed (see Table $1^{2}$ ) represents what we know about sources detected by ISGRI to date (until December 1, 2006). The structure of Table 1 is as follows:

\section{- Name:}

Most sources have more than 1 name owing to detections by various instruments operating at different energies. As in Ebisawa et al. (2003), we selected names that are commonly used in high-energy astrophysics and that are accepted as an identifier in SIMBAD. This eases comparisons with other catalogs.

- Position:

Source positions are from the X-rays unless a more

\footnotetext{
${ }^{2}$ Table 1 is only available in electronic form.
}

accurate position at other wavelengths is known for a confirmed counterpart. Right Ascension and Declination in J2000 coordinates are given in "hh mm ss.s" and "deg arcmin arcsec" formats, respectively. The uncertainty (in arcmin) of the position from the reference is given as an individual entry, and it is reflected in the representation of the source positions. Galactic coordinates are also provided.

- Absorption:

Column densities (in $10^{22} \mathrm{~cm}^{-2}$ ) were gathered from the literature whenever a model fit to the X-ray spectrum required an absorption component. Extracting a single $N_{\mathrm{H}}$ for a source and comparing this value to those of other sources is not a straightforward exercise since intrinsic column densities are not static. A measurement made during flaring or quiescent periods, or at different orbital epochs, will heavily influence the $N_{\mathrm{H}}$. The geometry of the system, the energy range of the satellite that gathered the data, and the model used to describe the resulting spectrum also affect the $N_{\mathrm{H}}$ value. Therefore, the uncertainties are often large or only upper limits are provided. Whenever possible, we selected the $N_{\mathrm{H}}$ value of the model that best fits a recent X-ray spectrum taken with a telescope that covers the soft X-ray domain well.

- Modulations:

Spin periods (in seconds) and/or orbital periods (in days) 
Table 2. The number of sources from each of the major classes detected by ISGRI are listed for new (झIGRs) and previously-known sources. Miscellaneous (Misc.) sources are Galactic objects that are not X-ray binaries (e.g. CVs, SNRs, AXPs, etc.) and Uncl. refers to the group of sources that have yet to be classified.

\begin{tabular}{lllllll}
\hline \hline & HMXBs & LMXBs & AGN & Misc. & Uncl. & Total \\
\hline IGRs & $32(15 \%)$ & $6(3 \%)$ & $50(23 \%)$ & $15(7 \%)$ & $111(52 \%)$ & 214 \\
previously-known & $46(16 \%)$ & $76(27 \%)$ & $113(40 \%)$ & $32(11 \%)$ & $18(6 \%)$ & 285 \\
\hline Total & $78(16 \%)$ & $82(16 \%)$ & $163(33 \%)$ & $47(9 \%)$ & $129(26 \%)$ & 499 \\
\hline
\end{tabular}

have been reported for a large number of Galactic objects detected by ISGRI. Some systems are known to spin down, so we selected the most recent value from $R X T E$ whenever possible, even though this level of precision is not needed for the purpose of our study. The spin period can refer to the spin of the NS in X-ray binaries, or to the spin of the White Dwarf in CVs. The catalogs of Liu et al. (2000) and Liu et al. (2001), and the systematic analysis of RXTE data by Wen et al. (2006) are among the main references of spin and orbital periods in this work.

- Distances:

The distances to extragalactic objects are given as a measure of the redshift (denoted by brackets). Objects in the Milky Way and Magellanic Clouds have distances in units of kpc. Opinions sometimes differ as to the distances of some Galactic sources. We favored distance measurements that were recent, precise and that were the least modeldependent. Even so, the distance uncertainties quoted in the literature can be large. The distance measurements are from, among others, White \& van Paradijs (1996), Grimm et al. (2002), Jonker \& Nelemans (2004), Bassani et al. (2006), Beckmann et al. (2006a), or from references in Liu et al. (2000) and Liu et al. (2001). Note that the distances in Grimm et al. (2002) sometimes represent an average over several competing distance estimates.

- Type:

Source classifications are based on the concensus opinion in the literature. Peculiar behaviour, such as quasi-periodic oscillations, transience, and Z-track or Atoll shapes, are also noted since they can help us distinguish between systems within the same class. If the source classification has not yet been confirmed, it is simply called "unclassified". The transient identification of a source is given by any of the following: 1) the label was assigned by its discoverers or by other authors (e.g. "Discovery of a new transient IGR J..."); 2) the source has not been detected by anyone else since its discovery announcement, e.g. it is not listed in the all-data, all-sky catalogs of Bird et al. 2006, 2007); 3) the source is detected only in mosaic images of a single or a few consecutive revolutions according to Bird et al. (2007), but not in their all-data mosaic images.

- References:

All parameters are referenced so that the reader can assess the methods that were used to determine the quoted values and uncertainties.

\section{Results}

\subsection{Spatial distribution}

Table 2 lists the major source populations detected by ISGRI that are either new (三IGRs) or that were previously known. ISGRI has discovered many new HMXBs and AGN, but their proportions relative to the other classes are similar to what was known before the launch of INTEGRAL. Only a few LMXBs have been discovered by ISGRI. This is because LMXBs are generally less intrinsically obscured than HMXBs or AGN, and are therefore easier to detect with previous satellites. Around 50 sources have been detected that belong to the group of miscellaneous sources (i.e. CVs, SNRs, PWN, AXPs, etc.), while 130 IGRs await classification.

The spatial distributions, in Galactic coordinates, of the major classes of $\gamma$-ray sources detected by ISGRI are presented in Fig. 1. Naturally, ISGRI detects sources in regions that are exposed. Given the heterogeneous exposure map of the sky gathered in the last 4 years of observations, detections are biased towards regions of the sky that have been exposed the longest (i.e. the Galactic plane and bulge).

However, Fig. 1 also demonstrates the effect that the evolution of each type of source has on its spatial distribution. Because their optical companions belong to an old stellar population, LMXBs are found predominantly in the Galactic bulge and/or they have had time to migrate off the plane of the Milky Way $\left(|b| \gtrsim 3-5^{\circ}\right)$. On the other hand, the stellar companions of HMXBs are young stars, so these systems must remain close to sites of recent stellar formation. Thus, the angular distribution of HMXBs reflects the spiral structure of the Galaxy, with an uneven distribution along the Galactic plane punctuated by peaks that are roughly consistent with the tangential directions to the inner spiral arms. Those HMXBs that have been detected at longitudes $|l| \gtrsim 90^{\circ}$ correspond to systems located around spiral arms near the Sun. Evolutionary signatures like these were noticed in the past by Ginga (Koyama et al. 1990), RXTE (Grimm et al. 2002), and more recently with INTEGRAL (Dean et al. 2005; Lutovinov et al. 2005), although their samples were smaller than the one presented here.

Another way to demonstrate the role of stellar evolution in shaping the spatial distributions of LMXBs and HMXBs is to plot the positions of sources whose distances are known on a spiral arm model of the Milky Way. Russeil (2003) developed the Galactic spiral arm model that we used. Their model is based on the locations of star-forming complexes that include groups of OB stars, molecular clouds, H II regions, and diffuse ionised gas. The locations of these complexes are derived from a variety of tracers such as $\mathrm{H} \alpha, \mathrm{CO}$, the radio continuum and absorption lines.

While the uncertainties on distances can be large, Fig. 2 shows that HMXBs tend to occupy the outer disk and arms where young stars are formed, whereas LMXBs are clustered near the bulge where old globular clusters reside. A histogram of galactocentric radii (Fig. 3) shows LMXBs peaked at the center and decreasing gradually, while HMXBs roughly follow the distributions from H II/CO surveys (Russeil 2003) which are underabundant in the central few kpc and peak at the spiral arms. According to a Kolmogorov-Smirnov (KS) test, the probability is less than $0.01 \%$ that the galactocentric distributions of LMXBs and HMXBs are statistically compatible.

The distribution of LMXBs in the central Galaxy suggests an association with the Galactic bar (Fig. 2). Low-mass X-ray 


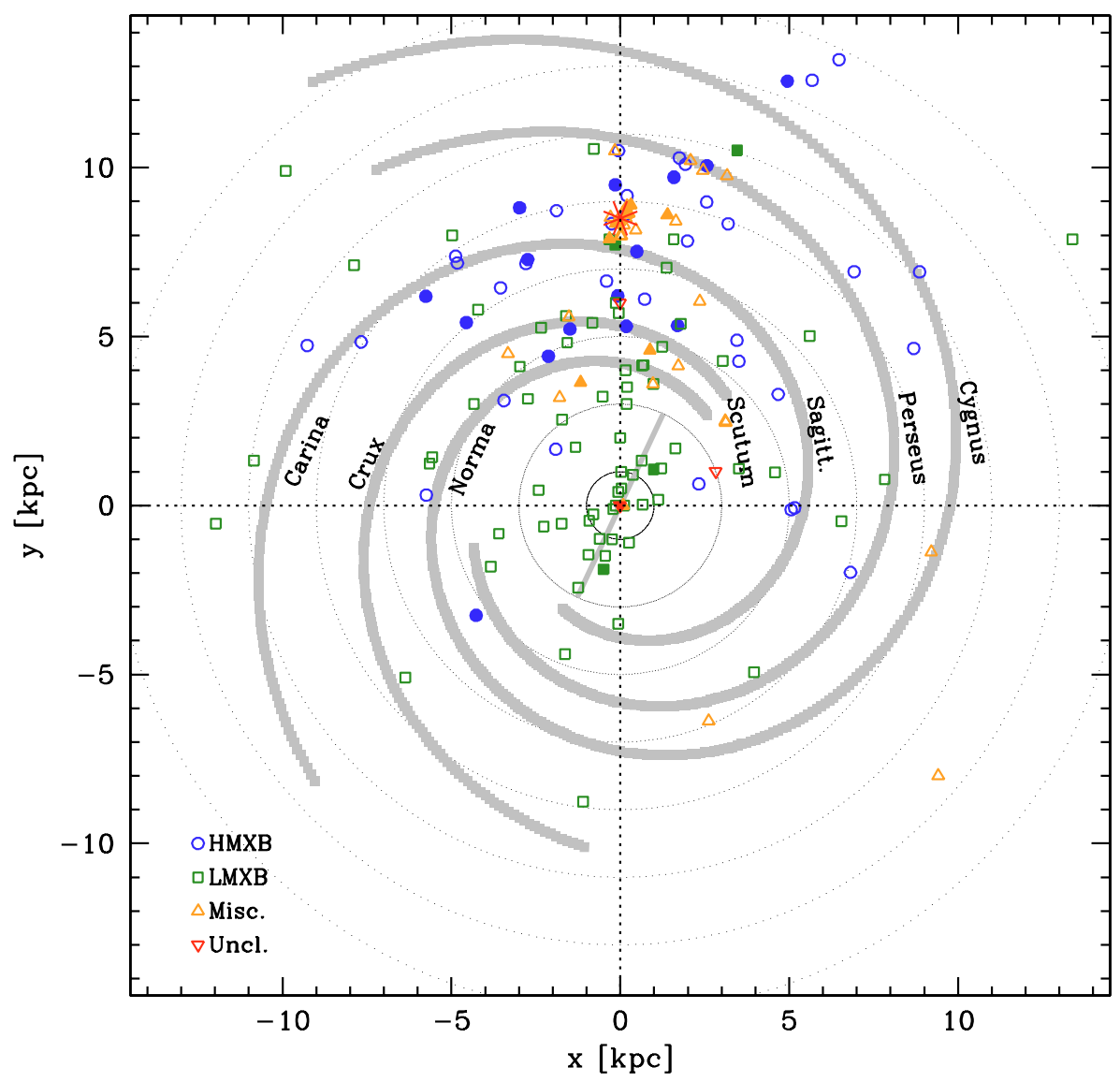

Fig. 2. Galactic distribution of HMXBs (49, circles), LMXBs (74, squares), miscellaneous sources (37, triangles), and unclassified sources (3, inverted triangles). Filled symbols represent IGR sources. Also plotted is the 4-arm Galactic spiral model from Russeil (2003) with the Sun located at $8.5 \mathrm{kpc}$ from the centre. The concentric circles indicate radii of $1,3,5, \ldots, \mathrm{kpc}$ from the centre.

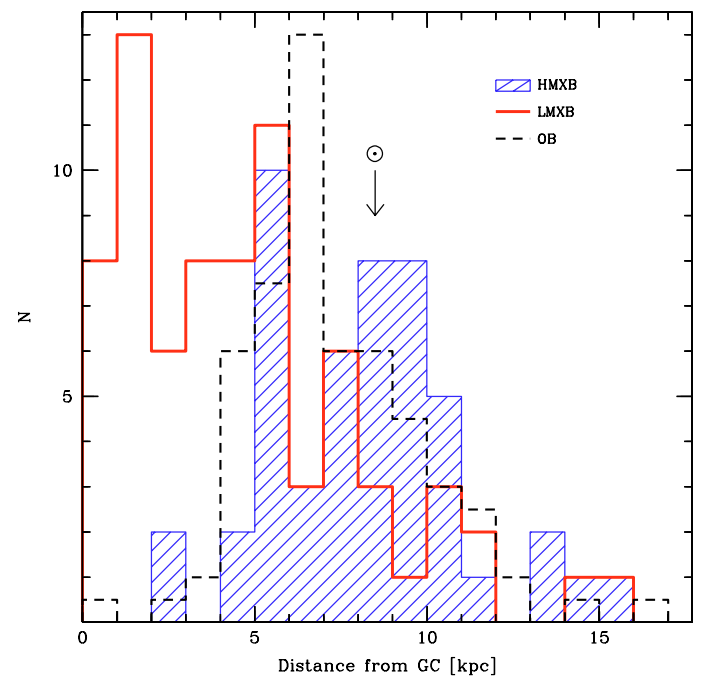

Fig. 3. Distribution of galactocentric distances of HMXBs (49, shaded histogram) and LMXBs (74, thick histogram). The dashed histogram represents OB star-forming complexes from Russeil (2003) (divided by 2 ).

binaries whose distances are known and that have been detected by ISGRI are not prevalent on either side of the bar. Only 1 LMXB with a distance measurement has been detected in the Galactic center region bound by $0<x<3 \mathrm{kpc}$ and $-3<y<0 \mathrm{kpc}$, indicating that the bar might be responsible for preventing an identification and distance measurement to be made for the faint counterparts to LMXBs situated behind it. As viewed from the Sun, the orientation of the bar leads to an apparent asymmetry of LMXBs in the central $3 \mathrm{kpc}$ of the

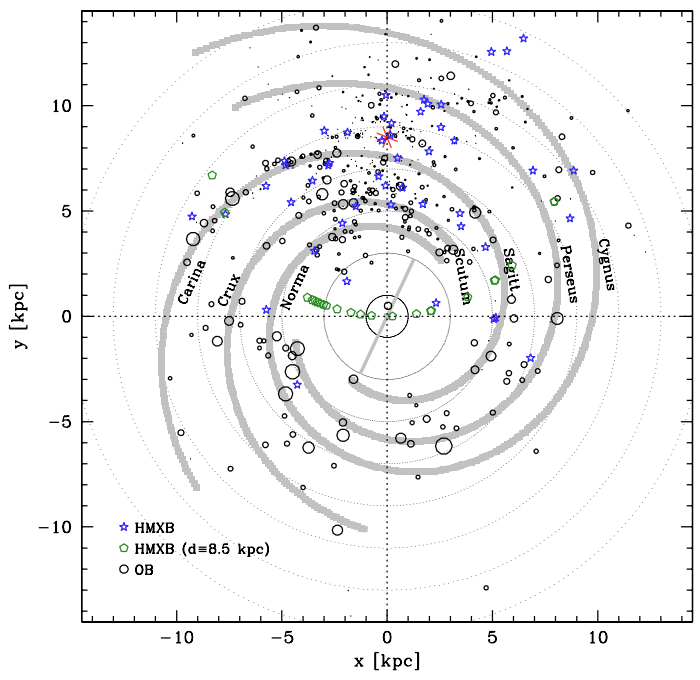

Fig. 4. Galactic distribution of HMXBs whose distance are known (49, star symbols) and the locations of star-forming complexes from Russeil (2003) (464, circles). The symbol size of the latter is proportional to the activity of the complex. HMXBs whose distances are unknown have been placed at $8.5 \mathrm{kpc}$ (23, pentagons).

Galaxy $\left(|l| \lesssim 20^{\circ}\right)$ : in this direction, ISGRI has detected $50 \%$ more LMXBs at negative longitudes than at positive longitudes. Maps of Galactic absorption are expected to be symmetrical in this region (Dickey \& Lockman 1990).

In Fig. 4, we present the Galactic distribution of star-forming complexes Russeil (2003) with the symbol size proportional to the excitation parameter in that region (三amount of ionising photons as determined from the radio continuum flux). High-mass 


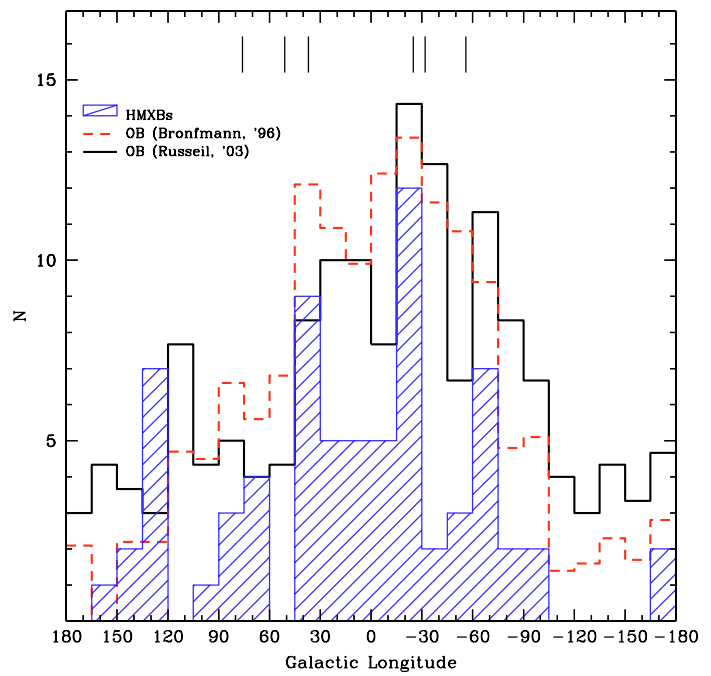

Fig. 5. Histograms of Galactic longitudes integrated over the latitude for HMXBs with $|b|<6^{\circ}$ (shaded histogram) and star-forming regions from Russeil (2003) (thick histogram, divided by 3 ) and from Bronfman et al. (1996) (dashed histogram, divided by 10). The vertical lines indicate the tangential directions of the 4-arm spiral model from Russeil (2003).

X-ray binaries whose distances are known are symbolised by stars, while those with unknown distances were assigned a distance of $8.5 \mathrm{kpc}$ and are represented by pentagons. The 4 -arm spiral model of Russeil (2003) is also drawn. Figure 5 presents histograms of Galactic longitudes (integrated over the latitude) of HMXBs (shaded histogram, with $|b|<6^{\circ}$, in order to exclude sources in the Magellanic Clouds). Also shown are angular distributions of star-forming complexes from Russeil (2003) (divided by 3 , thick histogram), and of ultra-compact H II regions detected by IRAS (Bronfman et al. 1996) (divided by 10, dashed histogram).

In general, the distribution of HMXBs along the plane of the Milky Way coincides with the expected radial distribution of young massive star-forming regions. A KS test yields a probability of $22 \%$ that the distributions of HMXBs and IRAS sources (shaded and dashed histograms, respectively, in Fig. 5) are statistically compatible. Excluding HMXBs that lie outside the survey region covered by Bronfman et al. (1996) $\left(|b|<2^{\circ}\right.$ for $|l|<60^{\circ}$, and $|b|<4^{\circ}$ elsewhere) increases the probability of statistical compatibility to $34 \%$. Peaks at Galactic longitudes $l \sim \pm 30^{\circ}$ are observed in both data sets corresponding to the direction of the inner spiral arm tangents (Norma and Scutum/Sagittarius arms). Bronfman et al. (1996) remark that the peaks in their distribution are also consistent with another active formation site of young, massive stars: a molecular ring situated at a radius of $\sim 3 \mathrm{kpc}$ from the Galactic center.

At first glance, the distributions of star-forming complexes of Russeil (2003) and HMXBs are also compatible (thick histogram in Fig. 5). The KS test returns a probability of only $3 \%$ which is misleading given the large number of objects in Russeil (2003) that are not very active. When selecting complexes with an excitation parameter $>10 \mathrm{pc} \mathrm{cm}^{-2}$, which still represents $70 \%$ of the sample, the statistical compatibility improves to $41 \%$.

Lutovinov et al. (2005) and Dean et al. (2005) found that the distribution of HMXBs was offset with respect to the directions of the spiral arm tangents. Lutovinov et al. (2005) note that $\sim 10$ Myr must elapse before one of the stars in a binary system collapses into a NS or $\mathrm{BH}$, and that Galactic rotation will induce changes in the apparent positions of the arms relative to the Sun.
This implies a delay between the epoch of star formation and the time when the number of HMXBs reaches its maximum. The observed displacement could simply stem from uncertainties in the distances to the HMXBs. Another problem is that the exact location of the arms depends on which Galactic model is used. Changes in the Sun-GC distance or in the pitch angles of the arms affects the radial scaling and shifts the tangential directions.

The propagation of density waves is believed to promote star formation in the spiral arms (Lin et al. 1969). Depending on the distance to the GC, the spiral arm pattern has angular velocities in the range of $\Omega \sim 20-60 \mathrm{Gyr}^{-1}$ (Bissantz et al. 2003). Hence, in the last $\sim 10 \mathrm{Myr}$ (corresponding roughly to the epoque when the inner spiral arms and the current density maxima of HMXBs overlapped), the inner arms of the Galaxy have rotated around the $\mathrm{GC}$ by $\sim 40^{\circ}$. Individual stars (such as the Sun) or groups of stars have negligible movement in this scenario. Instead, star-forming sites that are now active (e.g. from Russeil 2003) should be about $\sim 40^{\circ}$ away from those regions that were active some $10 \mathrm{Myr}$ ago and that produced the current crop of HMXBs. Therefore, in order to reproduce the distribution of active star-forming sites as they were some $10 \mathrm{Myr}$ ago, we introduced differential Galactic rotation to "unwind" the distribution of current star-forming complexes from Russeil (2003). Kolmogorov-Smirnov tests suggest that the effects of Galactic rotation are negligible, even when only the most active sites are considered.

The distribution of angular distances from the Galactic plane (in degrees, for $|b|<20^{\circ}$ ) of sources detected by ISGRI is shown in Fig. 6. The distributions were summed over the northern and southern Galactic hemispheres. Shaded histograms are HMXBs (left) and AGN (right), and the thick histograms represent LMXBs (left) and miscellaneous sources (right). The distribution of unclassified sources is given by the dashed histogram. Not surprisingly, the spread of the latitude distributions is larger in LMXBs than it is in HMXBs owing to the relative youth of the optical primaries in the latter. Also expected is the distribution of AGN which is more or less flat and which roughly follows the exposure map. However, the Galactic plane $\left(|b| \lesssim 3^{\circ}\right)$ is noticeably deficient in AGN detections despite the fact that the exposure map is biased here. This highlights the difficulty in detecting AGN at high energies and at low latitudes; these objects tend to be intrinsically absorbed, they are further obscured by the Galactic plane, and their counterparts have to be identified within a crowded field. Sazonov et al. (2007) noted that the exclusion of sources in the Galactic plane region $\left(|b|<5^{\circ}\right)$ from an all-sky survey resulted in only a marginal reduction in the number of identified AGN, whereas the number of unclassified sources dropped significantly.

It is useful to examine the unclassified sources as they help to define the limits of our study. Almost all of the sources that are unclassified have position accuracies that are no better than a few arcminutes. This precludes establishing an optical counterpart for many unclassified sources located in crowded regions such as the Galactic plane. The transient nature of many unclassified sources implies a lack of immediate follow-up observations that would permit a classification. Because of their transience, many unclassified sources appear fainter than average in longexposure mosaic images. Masetti et al. (2006) suggest that up to half of all unclassified sources could be AGN situated behind the Galactic plane, whereas Dean et al. (2005), working on a smaller sample, favor a Galactic origin for the unclassified sources of Bird et al. (2004) based on the slope of the $\log (N)-\log (S)$ relation and other factors. 


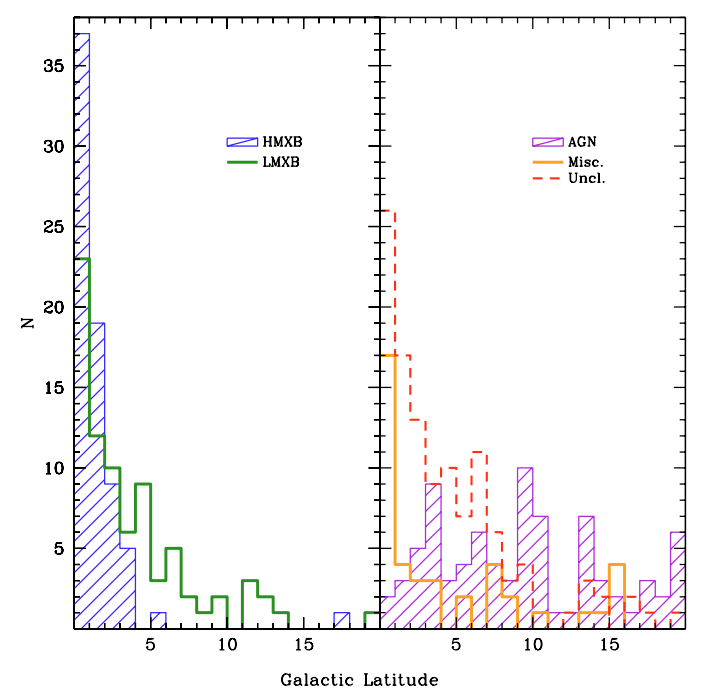

Fig. 6. Angular distribution (in degrees, for $|b|<20^{\circ}$ ) from the Galactic plane of sources that have been detected by ISGRI. Shaded histograms are HMXBs (left) and AGN (right), and the clear histograms represent LMXBs (left) and miscellaneous sources (right). The dashed histogram denotes unclassified sources. The distributions have been summed over the northern and southern Galactic hemispheres. The curves represent fits to the data from the model and parameters described in the text.

Unclassified sources have a distribution of Galactic latitudes that peaks in the central $3^{\circ}$ from the Galactic plane and decreases gradually, suggesting a population of sources that are Galactic rather than extragalactic in origin (see Fig. 6). Many of the unclassified sources also happen to be transient, whereas AGN can vary but tend to emit persistently. Furthermore, the angular distribution of unclassified sources is very similar to the distribution of LMXBs with a KS-test probability of nearly $40 \%$ of statistical compatibility between unclassifieds and LMXBs, compared with $13 \%$ for miscellaneous sources, and less than $0.01 \%$ for either AGN or HMXBs.

While there are extragalactic sources among them, the population of unclassified sources is therefore likely to be composed primarily of Galactic sources such as LMXBs and miscellaneous sources. We are unable to elaborate on the proportions of the different classes, but it is clear from Fig. 6, and from the results of KS tests, that the unclassified sources are most similar to the LMXBs and miscellaneous sources in their distribution off the Galactic plane. The reasons they have avoided classification (and detection by previous missions) are: the companion stars to LMXBs are usually faint in the optical/IR spectrum; they are located near the Galactic plane where absorption and source confusion prevent an identification; and, for many of these sources, their transient emission complicates efforts to perform followup observations. Recent improvements in Target of Opportunity campaigns aimed at new IGRs have uncovered as many new LMXBs in the last year than during the first 3 years of observations combined.

Figure 7 presents the distributions of scale heights (in kpc) for HMXBs (shaded histogram) and LMXBs (clear histogram) whose distances are known. Sources from the Magellanic Clouds are excluded. Following the procedure in Dean et al. (2005), we set the number of sources as a function of the distance in $\mathrm{kpc}$ above the Galactic plane $(h)$ according to $N=k \cdot \mathrm{e}^{-\alpha \cdot h}$ where $\alpha \equiv 1 / h_{0}$ describes the steepness of the exponential. The parameters that best fit this model are listed in Table 3 . The value that we derive for the characteristic scale height $\left(h_{0}\right)$ of HMXBs

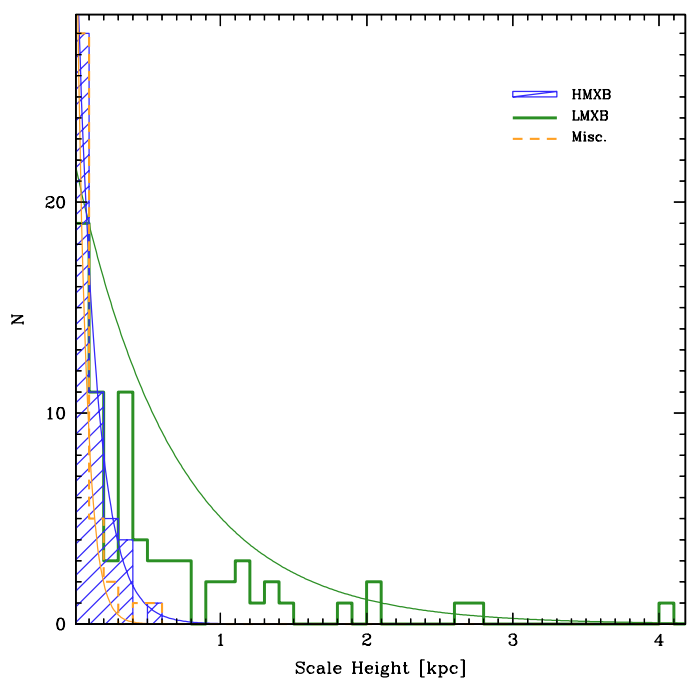

Fig. 7. Vertical scale height (in kpc) from the Galactic plane of HMXBs, LMXBs and miscellaneous sources whose distances are known. The distributions have been summed over the northern and southern Galactic hemispheres. Sources from the Magellanic Clouds are excluded. The curves represent the exponential model described in the text fit to the data (see Table 3 for parameters).

Table 3. Parameters from the model described in the text fit to the distributions of scale heights from the Galactic plane for HMXBs, LMXBs and miscellaneous sources whose distances are known. Objects from the Magellanic Clouds are excluded.

\begin{tabular}{lccc}
\hline \hline & $k$ & $\alpha$ & $h_{0}[\mathrm{pc}]$ \\
\hline HMXBs & $36 \pm 3$ & $7.5 \pm 1.7$ & $134_{-25}^{+39}$ \\
LMXBs & $22 \pm 3$ & $1.5 \pm 0.5$ & $680_{-160}^{+320}$ \\
Misc. & $41 \pm 3$ & $15 \pm 4$ & $66_{-14}^{+23}$ \\
\hline
\end{tabular}

is $\sim 130 \mathrm{pc}$ which is compatible with the value found by Grimm et al. (2002) with RXTE data, but slightly less than the value from Dean et al. (2005) ( $\gtrsim 200 \mathrm{pc})$. The characteristic scale height that we derive for LMXBs $(\sim 600 \mathrm{pc})$ is larger than the scale heights found by Grimm et al. (2002) and Dean et al. (2005) which were closer to $\sim 400 \mathrm{pc}$. This is probably due to the greater coverage of the sky and larger sample size of our study. Miscellaneous sources have a distribution that is more similar to HMXBs than it is to LMXBs.

\subsection{Absorption}

The column densities along the light of sight of some sources in our sample are higher than the value expected from radio maps (Dickey \& Lockman 1990) which implies absorbing material intrinsic to the source. On average, Galactic IGRs are more absorbed than the sources seen before INTEGRAL (by a factor of 4) with IGRs representing a sizable contingent of objects that have $N_{\mathrm{H}} \sim 10^{23} \mathrm{~cm}^{-2}$ (see Fig. 8). The average column density of sources that were previously known is $N_{\mathrm{H}}=1.2 \times 10^{22} \mathrm{~cm}^{-2}$ $(\sigma \sim 0.7)$ whereas IGRs have an average $N_{\mathrm{H}}=4.8 \times 10^{22} \mathrm{~cm}^{-2}$ $(\sigma \sim 0.6)$. A KS test yields a probability of less than $0.01 \%$ that the two distributions are statistically compatible.

The classified Galactic IGRs are mostly HMXBs (Table 2) which usually exhibit high column densities, either intrinsically due to the geometry of the system or extrinsically due to their location along the dusty Galactic plane. Note, however, that the 


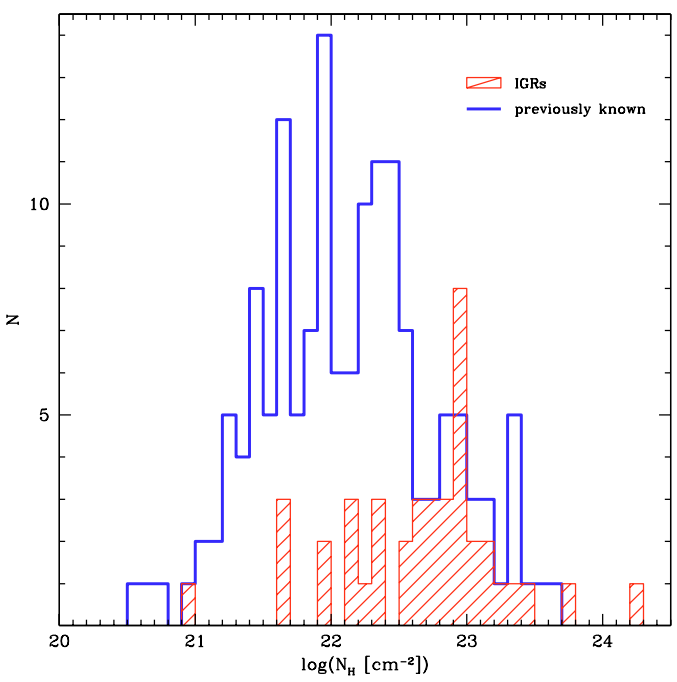

Fig. 8. The distribution of reported column densities $\left(N_{\mathrm{H}}\right)$ for Galactic sources (including sources in the Magellanic Clouds) detected by ISGRI that were previously known (152, clear histogram) and for IGRs (41, shaded histogram).

highest value of Galactic $N_{\mathrm{H}}$ is $\sim 3 \times 10^{22} \mathrm{~cm}^{-2}$ so objects with very large $N_{\mathrm{H}}$ can not be explained by interstellar absorption alone. Also keep in mind that the absorption from Dickey \& Lockman (1990) tends to be underestimated given that local small-scale inhomogeneities and the contribution from molecular hydrogen are ignored. The main reason that more absorbed sources are being found is that by operating above $20 \mathrm{keV}$, ISGRI is immune to the absorption that prevented their discovery with earlier soft X-ray telescopes. A large absorption is also a common feature of extragalactic IGRs. However, our data show that as a group, they are not more absorbed than pre-INTEGRAL AGN, in agreement with the conclusions of Beckmann et al. (2006a) and Sazonov et al. (2007).

Figure 9 presents an all-sky map of sources detected by ISGRI with symbol sizes proportional to reported column densities $\left(N_{\mathrm{H}}\right)$. Contours of expected line-of-sight absorption (Dickey $\&$ Lockman 1990) are provided for levels of $10^{21}, 5 \times 10^{21}$ and $10^{22} \mathrm{~cm}^{-2}$. One of the benefits of such a map is that potential clustering or asymmetries in the local distribution of matter can be studied. The lower portion of Fig. 9 shows that the Norma Arm region hosts many of the most heavily-absorbed Galactic sources $\left(N_{\mathrm{H}} \geq 10^{23} \mathrm{~cm}^{-2}\right)$ continuing a previously noted trend (e.g., Kuulkers 2005; Lutovinov et al. 2005; Walter et al. 2006). This region also happens to be the most active formation site of young supergiant stars (Bronfman et al. 1996). These stars are the precursors to the absorbed HMXBs that ISGRI is discovering in the Norma Arm. The Galactic Bulge and the Scutum/Sagittarius Arms are also represented by obscured sources but to a lesser extent than in the Norma Arm.

For sources whose distance are known, we did not find any clear dependence of the intrinsic $N_{\mathrm{H}}$ on the distance to the source, nor did we find a dependence of $N_{\mathrm{H}}$ with the luminosity as derived from the soft-band fluxes $(20-40 \mathrm{keV})$ listed in Bird et al. (2007).

\subsection{Modulations}

The strong magnetic fields in some NS X-ray binaries can produce non-spherically symmetric patterns of emission. If the magnetic and rotation axes are misaligned, this results in pulsations in the X-ray light curve.

Most IGRs for which a pulsation has been measured have spin periods $\left(P_{\mathrm{s}}\right)$ in the range of 100-1000 s, or around 10 times longer than the average pulse period of pre-INTEGRAL sources (Fig. 10). There are notable IGRs that represent extreme cases: IGR J00291+5934 has a pulse period of only 1.7 ms making it the fastest accretion-powered pulsar ever observed (Galloway et al. 2005), whereas IGR J16358-4726 has a spin period as long as $6000 \mathrm{~s}$ (Lutovinov et al. 2005; Patel et al. 2006). One of the reasons that IGRs have longer pulse periods than average is because many of them are SG HMXBs which are wind-fed systems with strong magnetic fields that tend to have the longest pulse periods (e.g. Corbet 1984). Another reason is that INTEGRAL and $X M M$-Newton feature long orbital periods around the Earth. This means that the source can be observed for long periods of time without interruptions, so that pulsations on the order of a few hundreds of seconds or more can be detected. Meanwhile, the previously-known sources in Fig. 10 include millisecond pulsars and other LMXBs, radio pulsars, CVs, etc., which are underrepresented among IGRs. To illustrate this, we performed a KS test which returned a very low probability $(0.0007 \%)$ of statistical compatibility between the distributions of 18 IGRs (shaded histogram) and 92 previously-known pulsars of all types (clear histogram) as they are presented in Fig. 10. The KS-test probability improved by an order of magnitude when IGRs were compared to 49 previously-known HMXBs, and it improved by 3 orders of magnitude when IGRs were compared to 14 previously-known SG HMXBs. So INTEGRAL is not just finding new pulsars that are HMXBs, but these HMXBs are predominantly long-period systems with SG companions.

The distribution of orbital periods $\left(P_{\mathrm{o}}\right)$ of IGRs exhibits a similar bimodal shape to that seen in the distribution of orbital periods known before INTEGRAL (Fig. 11). The probability of statistical compatibility is nearly $80 \%$ according to a KS test. The bimodal distribution represents 2 underlying populations: LMXBs (and miscellaneous sources) which tend to have short orbital periods, and HMXBs which tend to have longer orbital periods (Fig. 12).

In a Corbet $P_{\mathrm{s}}-P_{\mathrm{o}}$ diagram (Corbet 1984), members of each subclass of HMXBs segregate into different regions of the plot owing to the complex feedback processes between the modulation periods and the dominant accretion mechanism. Figure 13 shows that the majority of IGRs are located among other known SG HMXBs. The figure also shows that Be HMXBs have longer orbital periods than SG HMXBs, in general. While this fact was already known (e.g. Stella et al. 1986), the discrepancy remains even though INTEGRAL has nearly doubled the number of such systems.

\subsection{Modulations vs. absorption}

Accretion affects the spin period of a NS. If the velocity at the corotation radius (the radius at which the magnetic field regulates the motion of matter) exceeds the Keplerian velocity, then material will be spun away taking angular momentum with it and the NS will slow down due to the "propellor mechanism" (Illarionov \& Sunyaev 1975). For corotation velocities smaller than the Keplerian velocity, the material is able to accrete onto the NS magnetosphere which will either spin up or spin down the NS depending on whether the angular momentum of the accreted material has the same or an opposite direction as the NS spin (Waters \& van Kerkwijk 1989). So the spin rate of the pulsar in 


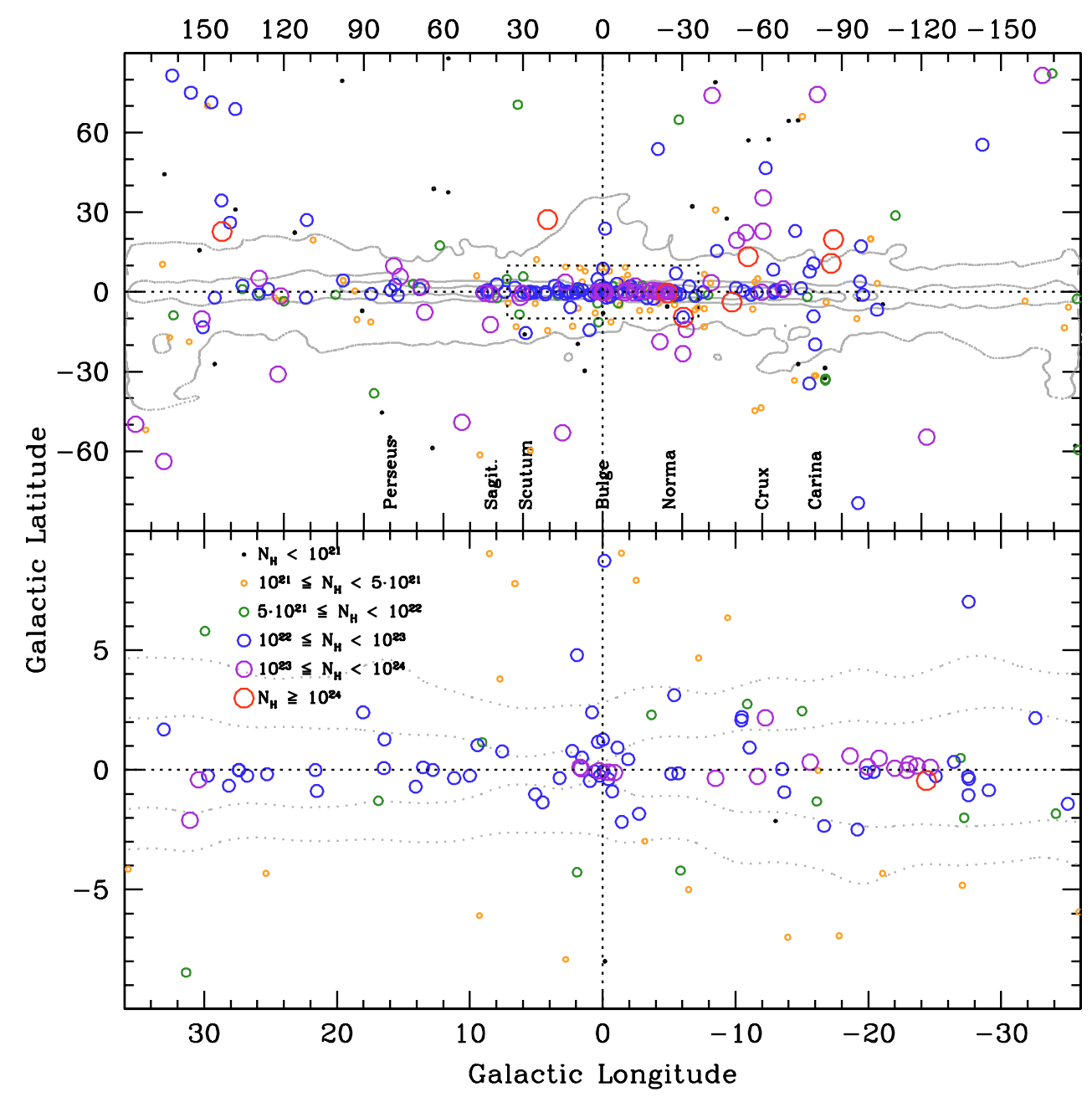

Fig. 9. Spatial distribution, in Galactic coordinates, of all sources detected by ISGRI for which $N_{\mathrm{H}}$ has been reported. The symbol size is proportional to the published column density. The figure at the top shows the whole sky and includes extragalactic sources, while the figure at the bottom focuses on the Bulge region (boxed region in the figure at the top) and excludes extragalactic sources. Contours denote Galactic absorption levels of $10^{21}, 5 \times 10^{21}$, and $10^{22} \mathrm{~cm}^{-2}$ (Dickey \& Lockman 1990).

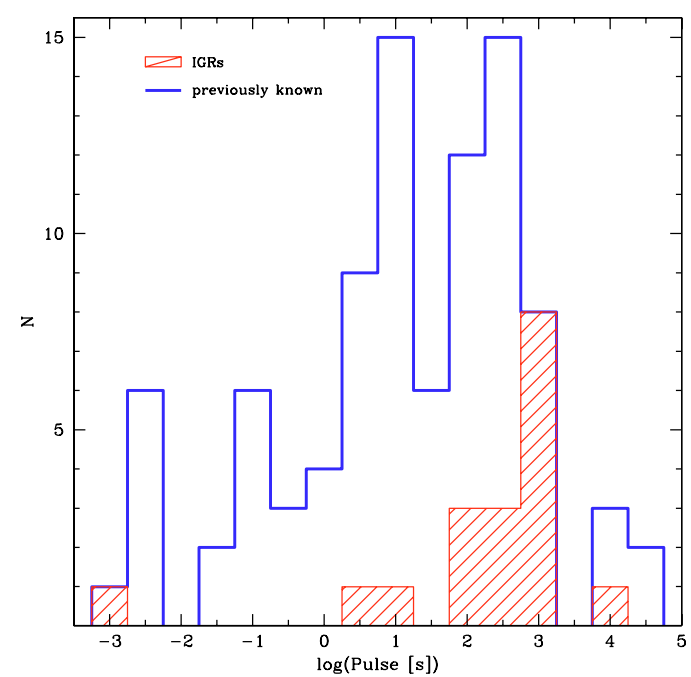

Fig. 10. Spin periods reported for sources detected by ISGRI that were previously known (92, clear histogram) and for IGRs (18, shaded histogram).

a HMXB is regulated by, among other things, the angular momentum of the wind of the stellar companion.

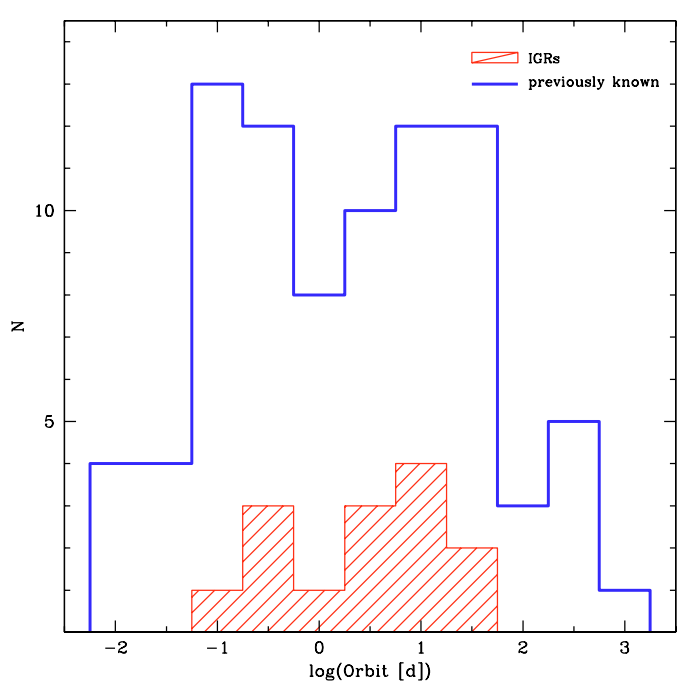

Fig. 11. Published orbital periods of sources detected by ISGRI. The clear histogram represents sources that were previously known (84) while the shaded histogram represents IGRs (14).

Assuming spherically-symmetric accretion from a radiationdriven wind of a SG star, the density of the wind as a function 


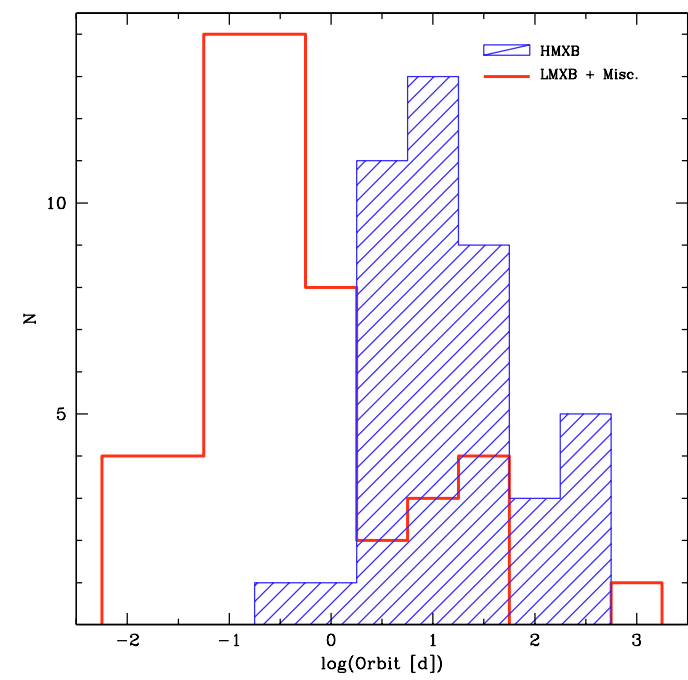

Fig. 12. Distribution of orbital periods of HMXBs (43, shaded histogram) compared with LMXBs and Miscellaneous sources (54, clear histogram).

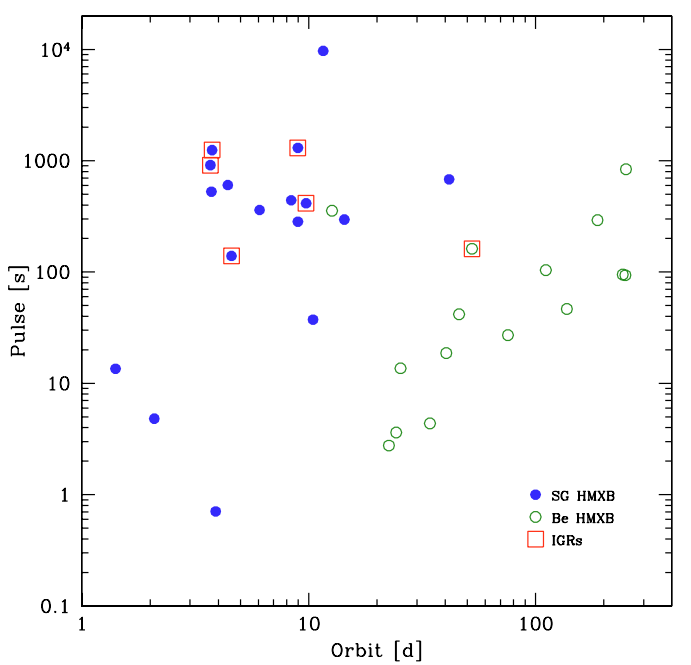

Fig. 13. Corbet diagram of spin vs. orbital period of HMXBs detected by ISGRI whose companions are OB supergiants (17, filled circles) or Be stars (15, empty circles). IGRs are boxed.

of radius is $\rho(r) \propto r^{-2}$. On the other hand, the structure of the winds of Be stars is believed to consist of dense slow equatorial outflows and thin fast polar winds (Lamers \& Waters 1987). The density drops much faster with the radius $\left(\rho(r) \propto r^{-3}\right)$ (Waters et al. 1988). Therefore, the winds of Be stars present stronger density and velocity gradients inside the capture radius of the NS, in both radial and azimuthal directions, which suggests that wind-fed accretion is more efficient at delivering angular momentum to the NS in Be HMXBs than it is in SG HMXBs (Waters \& van Kerkwijk 1989).

Given the density structures described above, and assuming a steady accretion rate of material whose angular momentum has the same direction as the spin of the NS, the spin period of the NS will reach an equilibrium value $P_{\text {eq }} \propto \rho^{-3 / 7}$. However, the present-day spin periods of NS in SG systems are much longer than predicted and are actually closer to $P_{\text {eq }}$ of the stellar winds while the star was still on the MS (Waters \& van Kerkwijk 1989). The equilibrium spin period in Be systems is constantly adjusting to the changing conditions in the winds (Waters \& van Kerkwijk 1989). As with the SG systems, pulsars in

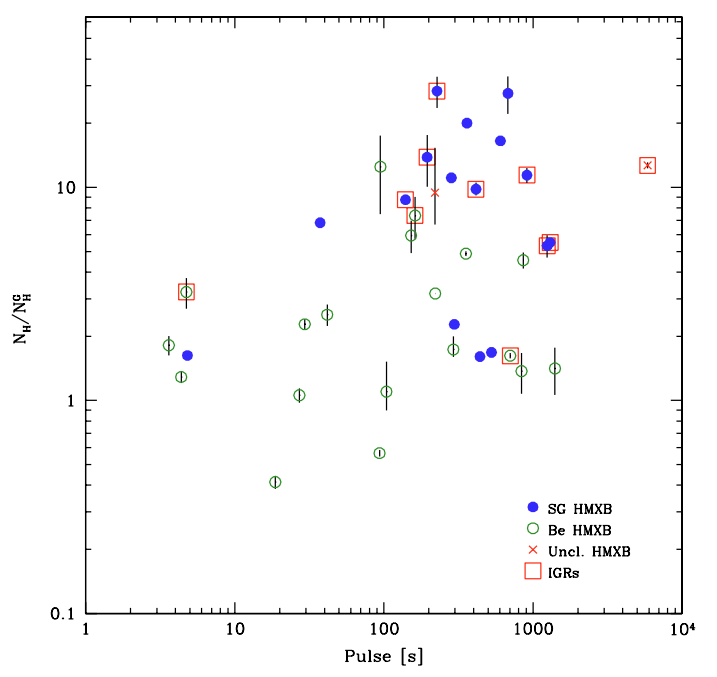

Fig. 14. Spin period as a function of reported $N_{\mathrm{H}}$ value (normalised by the expected Galactic value from (Dickey \& Lockman 1990)) for HMXBs detected by ISGRI whose companions are OB supergiants (16, filled circles), Be stars (19, empty circles), or unclassified (2, crosses). IGRs are boxed and Magellanic Cloud sources are excluded.

Be systems are not currently spinning at $P_{\text {eq }}$ but reflect the values of an earlier evolutionary stage (King 1991). So even though the transport of positive angular momentum through the wind is so inefficient that it can not spin up the pulsar to its expected equilibrium spin period, this does not influence how well the pulsar can be spun down by the "propellor mechanism" (Waters \& van Kerkwijk 1989).

With a few exceptions, HMXBs from the Milky Way that have been detected by ISGRI are segregated into distinct regions of a $P_{\mathrm{s}}-N_{\mathrm{H}}$ diagram (Fig. 14) stemming from the higher average $N_{\mathrm{H}}$ and longer average $P_{\mathrm{s}}$ of SG HMXBs compared to Be HMXBs. The SG HMXBs set apart from the others $\left(P_{\mathrm{s}}<\right.$ $50 \mathrm{~s})$ are Cen $\mathrm{X}-3$ which is a Roche-lobe overflow system, and OAO 1657-415 which might be transitioning from a wind-fed to a disk-fed system (Audley et al. 2006). The $N_{\mathrm{H}}$ values of sources in Fig. 14 have been normalised by the line-of-sight values $\left(N_{\mathrm{H}}^{\mathrm{G}}\right)$ from Dickey \& Lockman (1990). This normalisation does not affect our conclusions but it helps to reduce the scatter in the vertical direction, particularly for nearby sources such as X Per.

There could be a weak positive correlation between the $N_{\mathrm{H}}$ and spin period for HMXBs as a group. There are no highlyabsorbed sources $\left(N_{\mathrm{H}}>10^{23} \mathrm{~cm}^{-2}\right)$ with spin periods shorter than a few tens of seconds, and there are no pulsars with $P_{\mathrm{s}}>$ $100 \mathrm{~s}$ that are poorly absorbed $\left(N_{\mathrm{H}}<10^{22} \mathrm{~cm}^{-2}\right)$. A leastsquares fit to the data yields $P_{\mathrm{S}} \propto N_{\mathrm{H}}^{5 / 7}$. If we consider the $N_{\mathrm{H}}$ to be a reliable estimate of the density of matter around the compact object, then the slope that we find contradicts the slope expected from the equilibrium values ( -3/7). However, as noted above, the pulsars in Fig. 14 are spinning at periods that are longer than their equilibrium values would suggest.

Since Be HMXBs tend to have longer orbital periods than SG HMXBs (see Fig. 13), a distinction is also seen among the distributions of the $N_{\mathrm{H}}$ values and orbital periods of HMXBs with Be or SG companions (Fig. 15). There also appears to be an anti-correlation of $N_{\mathrm{H}}$ and orbital period: a least-squares fit to the data returns $P_{\mathrm{o}} \propto N_{\mathrm{H}}^{-3 / 7}$. In both types of systems, a shorter orbital period implies a compact object that is embedded deeper or spends more time in the dense regions of its stellar companion's wind resulting in more absorption. Therefore, Be HMXBs 


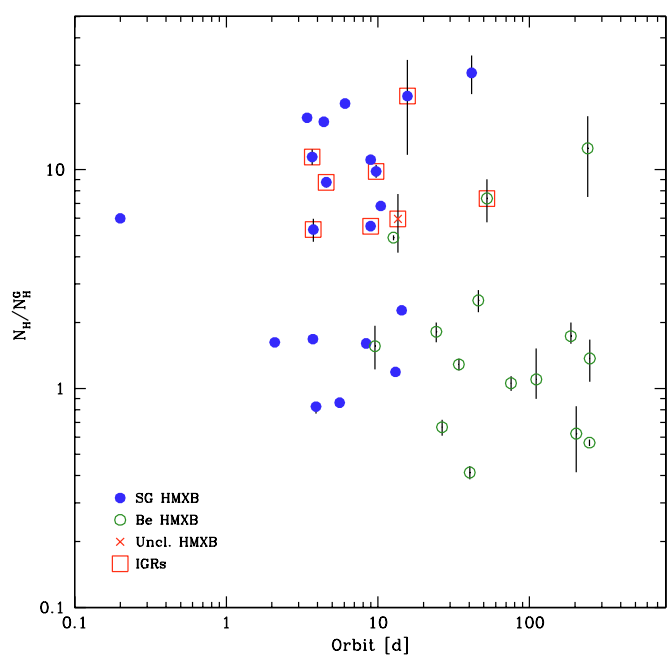

Fig. 15. Orbital period versus reported $N_{\mathrm{H}}$ value (normalised by the expected Galactic value from (Dickey \& Lockman 1990)) for HMXBs detected by ISGRI whose companions are OB supergiants (20, filled circles), Be stars (15, empty circles) or unclassified (1, cross). IGRs are boxed and Magellanic Cloud sources are excluded.

continue the trend set by SG HMXBs into long-orbital periodicity and low $-N_{\mathrm{H}}$ regions of the plot.

Spearman rank tests to the $P_{\mathrm{s}}-N_{\mathrm{H}}$ and $P_{\mathrm{o}}-N_{\mathrm{H}}$ distributions return weak positive and negative correlations with coefficients of 0.37 and -0.33 , respectively, suggesting that the null hypothesis of mutual independence between $N_{\mathrm{H}}$ and $P_{\mathrm{S}}$ or $P_{\mathrm{o}}$ can be rejected. From Monte Carlo simulations, we determined that the probability of finding a Spearman rank coefficient $\gtrsim 0.33$ is around 5\%. Admittedly, the scatter in the data is large as can be seen in Figs. 14-15. Because there are large uncertainties in the $N_{\mathrm{H}}$ and practically no uncertainty in the spin and orbital periods, the slope from a least-squares fit will tend to overestimate the real slope. Futhermore, the conclusions that we derive for how divergent species of objects react to changes in the local absorbing matter are based on a simplification of the underlying physics. The inclinations of the systems and their eccentricities, for example, are ignored. Even if we can not fit a slope of $-3 / 7$ to the data in Fig. 14, the correlations that we find in Figs. 14-15 might simply be due to the segregation of the 2 populations into distinct regions of the plots, rather than being due to physical processes.

Nevertheless, as more sources are added to these diagrams, the potential trends that have emerged could help constrain models describing the influence of local absorbing matter on the modulations. Another advantage of these plots is that the probable designation of an unidentified source is much more likely to be correct than when only a single parameter is used. This is illustrated in Fig. 16 where distributions of the 3 parameters in question $\left(N_{\mathrm{H}}, P_{\mathrm{s}}\right.$ and $\left.P_{\mathrm{o}}\right)$ are presented for $\mathrm{SG}$ and Be HMXBs. Other than in the orbital periods, and in the extremities of the $N_{\mathrm{H}}$ and $P_{\mathrm{s}}$ distributions, there is little that differentiates the 2 groups. A HMXB selected from an average bin in either $N_{\mathrm{H}}$ or $P_{\mathrm{S}}$ has a roughly equal probability of hosting a $\mathrm{SG}$ or Be star. However, a random HMXB in the $N_{\mathrm{H}}-P_{\mathrm{s}}$ plot will tend to be located among other members of its group.

Therefore, these diagrams could serve as new tools to help distinguish between SG and Be HMXBs when only $N_{\mathrm{H}}$ and either the spin or orbital periods are known. For example, IGR J19140+0951 has an orbital period of around 13 days and $N_{\mathrm{H}} \sim 10^{23} \mathrm{~cm}^{-2}$. It is positioned among other SG HMXBs so
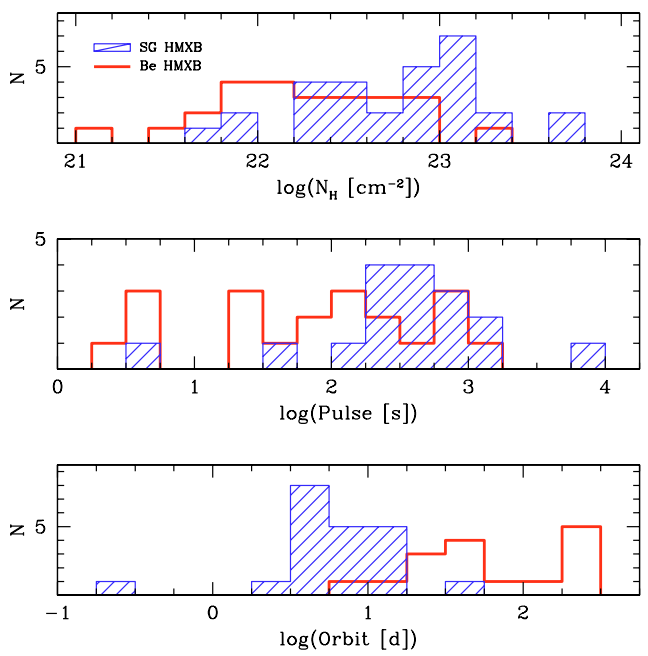

Fig. 16. Distribution of reported $N_{\mathrm{H}}$ (top), pulse periods (middle), and orbital periods (bottom) among HMXBs detected by ISGRI whose companions are OB supergiants (shaded histograms) or Be stars (clear histograms). Magellanic Cloud sources are excluded.

its companion is probably an OB supergiant (boxed cross in Fig. 15). This designation has already been suggested based on other criteria such as the source's persistent emission (Rodriguez et al. 2005). Recent IR observations of the source indicate a spectral type of B0.5-Ia which confirms the supergiant nature of the companion (D. Hannikainen, private communication). Similarly, AX J1749.2-2725 which is currently an unclassified HMXB could have a SG companion based on its position in Fig. 14 $\left(N_{\mathrm{H}}>10^{23} \mathrm{~cm}^{-2}\right.$ and pulse period over $\left.100 \mathrm{~s}\right)$. The other unclassified HMXB in Fig. 14 is IGR J16358-4726, whose NS has an unusually long spin period suggesting a magnetar nature for the source (Patel et al. 2006). This object is clearly in the SG HMXB camp based on its position in the plot.

\section{Summary and conclusions}

We have compiled a catalogue of all $\sim 500$ sources that were detected by ISGRI during its first 4 years of observations. This includes published parameters such as positions, column densities, spin and orbital periods, and distances or redshifts. The primary aims of this catalogue were to gather in a single place the most important parameters of high-energy sources detected by ISGRI and to use this large sample to test against various theoretical predictions, to search for possible trends in the data, and to determine where new sources fit in the parameter space established by previously-known high-energy sources.

Clustered towards the spiral arm tangents and at low Galactic latitudes, HMXBs follow the distributions of tracers of starforming regions. In contrast, most LMXBs are found in the Galactic bulge or have had time to migrate to high latitudes, typical of an older stellar population. The discrepancy is seen again in galactocentric profiles where the number of LMXBs gradually decreases from its maximum in the central kpcs, while HMXBs avoid the central kpcs and are overrepresented at the peaks of $\mathrm{H} \mathrm{II} / \mathrm{CO}$ distributions.

Over 200 new sources have been discovered by ISGRI but many of them remain unclassified. Although some may be AGN behind the plane, unclassified sources have a spatial distribution that resembles a Galactic population (notably LMXBs and $\mathrm{CVs}$ ) rather than an extragalactic one. If the unclassified sources are composed primarily of LMXBs, as their spatial distributions 
and the transient emission of most them seem to suggest, then the reason they remain unclassified is because the faint optical/IR counterparts of such sources are difficult to identify in the crowded and obscure Galactic plane.

Since it operates above $20 \mathrm{keV}$ and is unhindered by absorption, ISGRI is discovering many new HMXBs and AGN that are intrinsically absorbed $\left(N_{\mathrm{H}} \sim 10^{22}-10^{24} \mathrm{~cm}^{-2}\right)$. On average, Galactic IGRs are more absorbed (by a factor of $\sim 4$ ) than sources that were previously known.

Spin periods for most IGR pulsars are between a few hundred to a few thousand seconds or somewhat longer than the average spin periods of sources known before INTEGRAL. The distribution of orbital periods for IGRs closely resembles the bimodal distribution set by previously-known sources. The peaks correspond to 2 underlying populations: LMXBs and miscellaneous sources such as CVs and SNRs which tend to have short orbital periods, and HMXBs which have longer orbital periods, in general. Almost all IGRs for which both spin and orbital periods have been measured are located in the region of wind-fed accretion in the Corbet diagram. This is a testament to the number of new SG HMXBs that INTEGRAL has discovered.

Thanks to the larger sample size of these new SG HMXBs, we were able to test for dependences of the spin and orbital periods of HMXBs on the amount of absorbing matter local to the source. While scatter is an issue, there is a clear segregation of HMXBs in both plots which could be used to help assign Be or SG sompanions to sources that are still unclassified. There could be trends in both the $P_{\mathrm{s}}-N_{\mathrm{H}}$ and $P_{\mathrm{o}}-N_{\mathrm{H}}$ diagrams. The possible correlation of $P_{\mathrm{s}} \propto N_{\mathrm{H}}^{5 / 7}$ appears to contradict the expected slope (-3/7, e.g. (Corbet 1984)) which confirms that current spin periods are longer than the predicted equilibrium values, and that the spin-up of the pulsar via the wind is not as effective as the spin-down via the "propellor mechanism". The potential anti-correlation in the $P_{\mathrm{o}}-N_{\mathrm{H}}$ plot means that the average column density varies inversely with the distance between the objects as one would expect. However, intrinsic absorption values can change and the potential trends we see, rather than being due to physical processes that make the parameters interdependent, could simply be the result of 2 populations of sources occupying different parameter spaces, i.e. SG HMXBs are generally more absorbed, they spin slower, and they have shorter orbits than Be HMXBs. Nevertheless, given $N_{\mathrm{H}}$ and either $P_{\mathrm{s}}$ or $P_{\mathrm{o}}$ for a HMXB, improves the chances of correctly predicting the type of counterpart it has, compared with relying on only a single parameter. Of course, confirmation of the spectral type of the donor star in a HMXB still requires an optical/IR observation.

This work takes advantage of multi-wavelength observations in order to understand the nature of IGRs, and to help clarify the mechanisms that govern each type of source. Among the challenges facing more detailed population studies is the limited sample size of each subclass. This can only be alleviated by using large-FOV instruments such as INTEGRAL to search for new sources, and by regularly observing each new source in other wavelengths so that the accumulation of evidence rules out all but a single type of object. Many of the new sources which have been classified are absorbed HMXBs with supergiant companions. The increasing number of these systems discovered by INTEGRAL could alter our view of the Galactic population of hard X-ray sources and the evolutionary scenarios of their massive stellar companions.

Acknowledgements. The authors thank the anonymous referee for their prompt review of the paper. A.B. thanks S.E. Shaw, S. Paltani and M. Türler for their input and discussions. A.B. also thanks R. Walter for useful discussions on the Galactic distribution of HMXBs. J.R. warmly thanks P. Laurent, A. Goldwurm and C. Gouiffes for a careful reading of the manuscript and useful comments. This publication uses observations obtained with the ESA science mission INTEGRAL. The INTEGRAL instrument and data centres were directly funded by ESA member states and the USA (NASA). This research has made use of: the SIMBAD database operated at CDS, Strasbourg, France; NASA's Astrophysics Data System Bibliographic Services; data obtained from the High Energy Astrophysics Science Archive Research Center (HEASARC) provided by NASA's Goddard Space Flight Center.

\section{References}

Audley, M. D., Nagase, F., Mitsuda, K., Angelini, L., \& Kelley, R. L. 2006, MNRAS, 367, 1147

Bassani, L., Molina, M., Malizia, A., et al. 2006, ApJ, 636, L65 Beckmann, V., Gehrels, N., Shrader, C. R., \& Soldi, S. 2006a, ApJ, 638, 642 Beckmann, V., Soldi, S., Shrader, C. R., Gehrels, N., \& Produit, N. 2006b, ApJ, 652,126

Bird, A. J., Barlow, E. J., Bassani, L., et al. 2004, ApJ, 607, L33

Bird, A. J., Barlow, E. J., Bassani, L., et al. 2006, ApJ, 636, 765

Bird, A. J., Malizia, A., Bazzano, A., et al. 2007, ApJ, in press [arXiv: astro-ph/0611493]

Bissantz, N., Englmaier, P., \& Gerhard, O. 2003, MNRAS, 340, 949 Bodaghee, A., Walter, R., Zurita Heras, J. A., et al. 2006, A\&A, 447, 1027

Bronfman, L., Nyman, L.-A., \& May, J. 1996, A\&AS, 115, 81

Corbet, R. H. D. 1984, A\&A, 141, 91

Courvoisier, T. J.-L., Walter, R., Rodriguez, J., Bouchet, L., \& Lutovinov, A. A. 2003, IAU Circ., 8063, 3

Dean, A. J., Bazzano, A., Hill, A. B., et al. 2005, A\&A, 443, 485

Dickey, J. M., \& Lockman, F. J. 1990, ARA\&A, 28, 215

Ebisawa, K., Bourban, G., Bodaghee, A., Mowlavi, N., \& Courvoisier, T. J.-L. 2003, A\&A, 411, L59

Forman, W., Jones, C., Cominsky, L., et al. 1978, ApJS, 38, 357

Galloway, D. K., Markwardt, C. B., Morgan, E. H., Chakrabarty, D., \& Strohmayer, T. E. 2005, ApJ, 622, L45

Grimm, H.-J., Gilfanov, M., \& Sunyaev, R. 2002, A\&A, 391, 923

Illarionov, A. F., \& Sunyaev, R. A. 1975, A\&A, 39, 185

Jonker, P. G., \& Nelemans, G. 2004, MNRAS, 354, 355

King, A. R. 1991, MNRAS, 250, 3P

Koyama, K., Kawada, M., Kunieda, H., Tawara, Y., \& Takeuchi, Y. 1990, Nature, 343,148

Kuulkers, E. 2005, in Interacting Binaries: Accretion, Evolution, and Outcomes, ed. L. Burderi, L. A. Antonelli, F. D'Antona, T. di Salvo, G. L. Israel, L. Piersanti, A. Tornambè, \& O. Straniero, AIP Conf. Proc., 797, 402

Lamers, H. J. G. L. M., \& Waters, L. B. F. M. 1987, A\&A, 182, 80

Lebrun, F., Leray, J. P., Lavocat, P., et al. 2003, A\&A, 411, L141

Lebrun, F., Terrier, R., Bazzano, A., et al. 2004, Nature, 428, 293

Lin, C. C., Yuan, C., \& Shu, F. H. 1969, ApJ, 155, 721

Liu, Q. Z., van Paradijs, J., \& van den Heuvel, E. P. J. 2000, A\&AS, 147, 25

Liu, Q. Z., van Paradijs, J., \& van den Heuvel, E. P. J. 2001, A\&A, 368, 1021

Lutovinov, A., Revnivtsev, M., Gilfanov, M., et al. 2005, A\&A, 444, 821

Masetti, N., Morelli, L., Palazzi, E., et al. 2006, A\&A, 459, 21

Patel, S. K., Zurita, J., Del Santo, M., et al. 2006, ApJ, in press [arXiv: astro-ph/0610768]

Rodriguez, J., Bodaghee, A., Kaaret, P., et al. 2006, MNRAS, 366, 274

Rodriguez, J., Cabanac, C., Hannikainen, D. C., et al. 2005, A\&A, 432, 235

Russeil, D. 2003, A\&A, 397, 133

Sazonov, S., Revnivtsev, M., Krivonos, R., Churazov, E., \& Sunyaev, R. 2007, A\&A, 462, 57

Sguera, V., Barlow, E. J., Bird, A. J., et al. 2005, A\&A, 444, 221

Stella, L., White, N. E., \& Rosner, R. 1986, ApJ, 308, 669

Stephen, J. B., Bassani, L., Malizia, A., et al. 2006, A\&A, 445, 869

Ubertini, P., Lebrun, F., Di Cocco, G., et al. 2003, A\&A, 411, L131

Walter, R., Courvoisier, T. J.-L., Foschini, L., et al. 2004, in The INTEGRAL Universe. Proceedings of the Fifth INTEGRAL Workshop, 1620 February 2004, Munich, Germany, ed. B. Battrick, Scientific ed. V. Schoenfelder, G. Lichti, \& C. Winkler (Noordwijk: ESA Publication Division), ESA SP-552, 417

Walter, R., Zurita Heras, J., Bassani, L., et al. 2006, A\&A, 453, 133

Waters, L. B. F. M., van den Heuvel, E. P. J., Taylor, A. R., Habets, G. M. H. J., \& Persi, P. 1988, A\&A, 198, 200

Waters, L. B. F. M., \& van Kerkwijk, M. H. 1989, A\&A, 223, 196

Wen, L., Levine, A. M., Corbet, R. H. D., \& Bradt, H. V. 2006, ApJS, 163, 372

White, N. E., \& van Paradijs, J. 1996, ApJ, 473, L25

Winkler, C., Courvoisier, T. J.-L., Di Cocco, G., et al. 2003, A\&A, 411, L1

Zurita Heras, J. A., de Cesare, G., Walter, R., et al. 2006, A\&A, 448, 261 
A. Bodaghee et al.: A description of INTEGRAL sources, Online Material $p 1$

\section{Online Material}




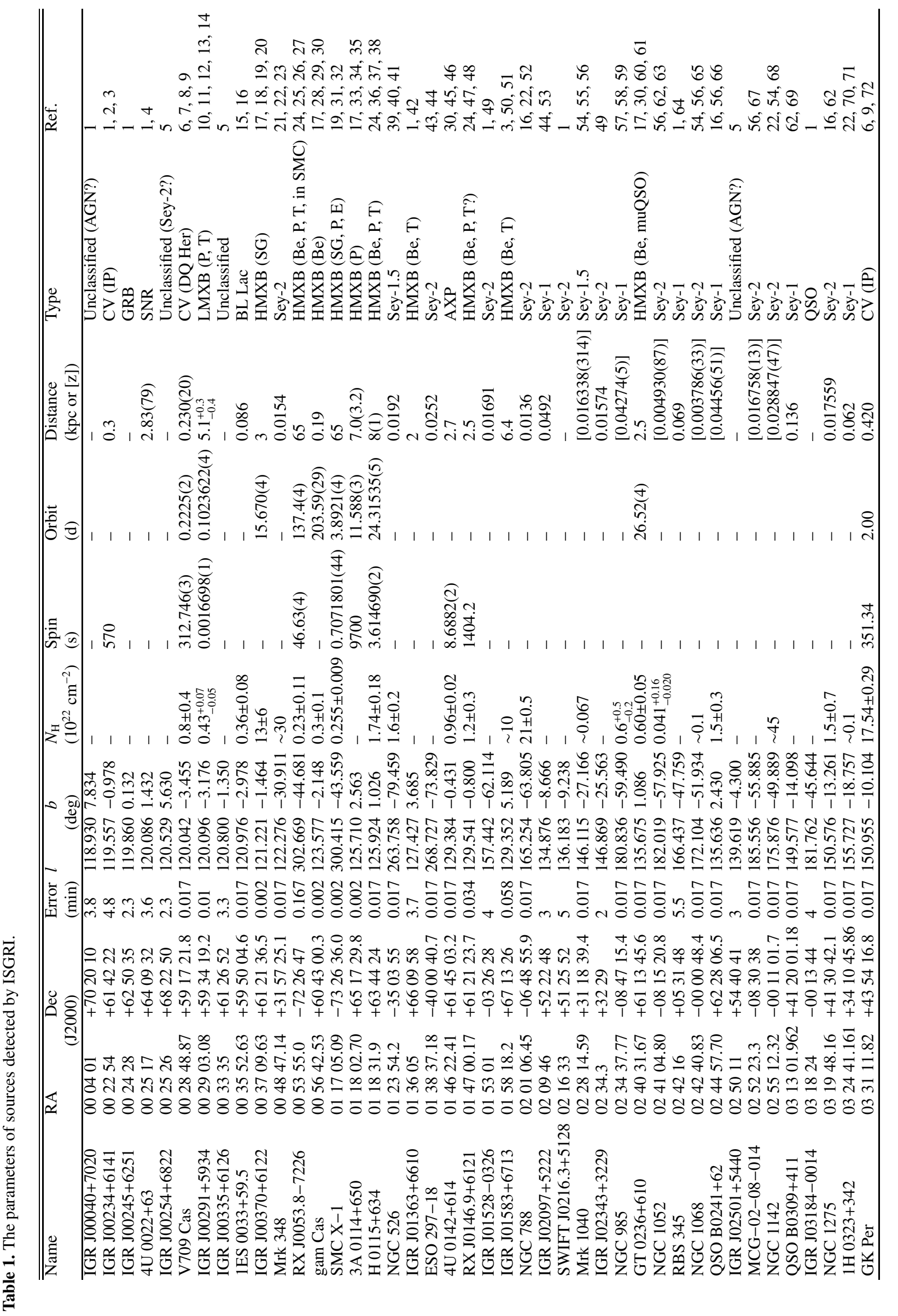




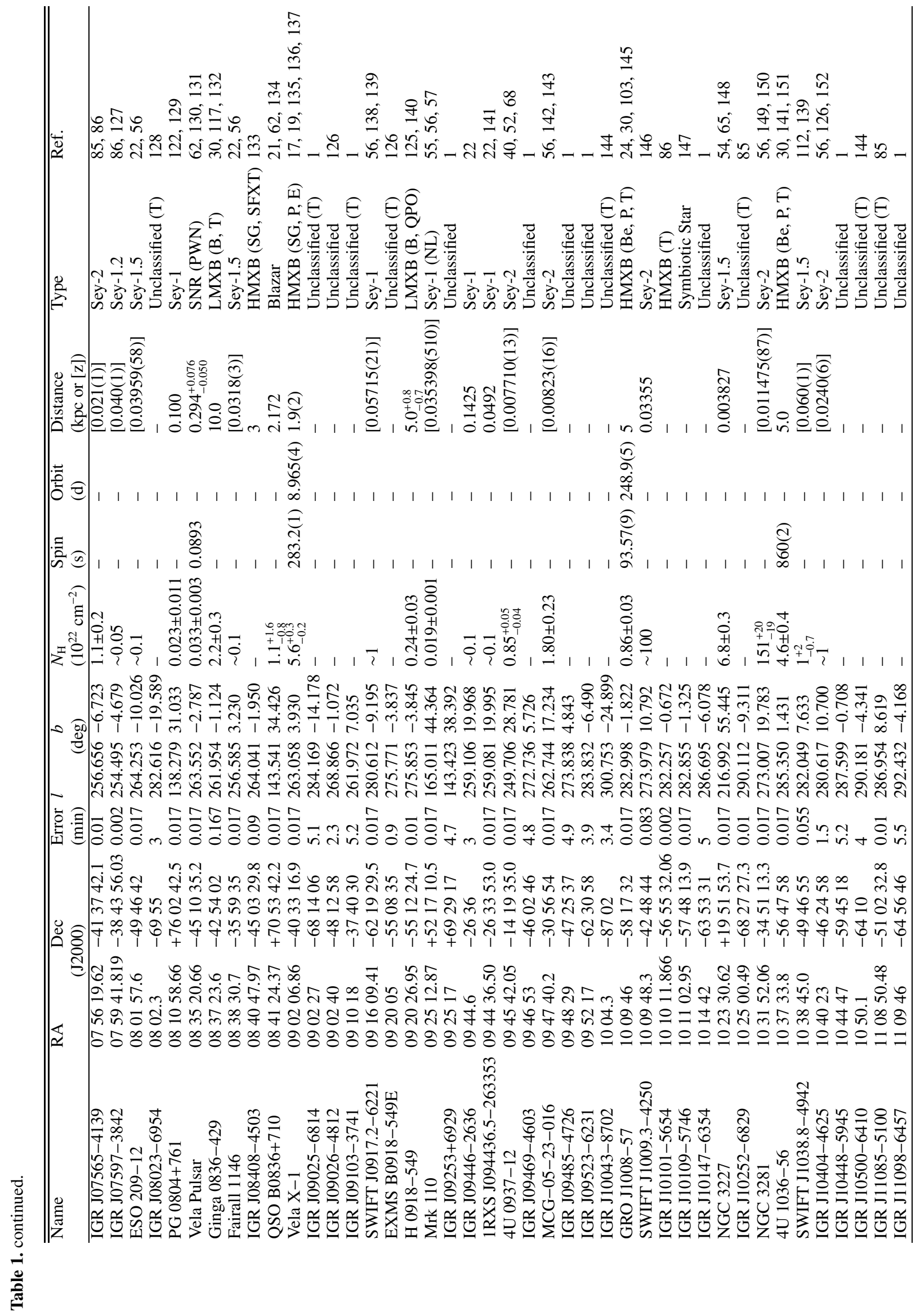




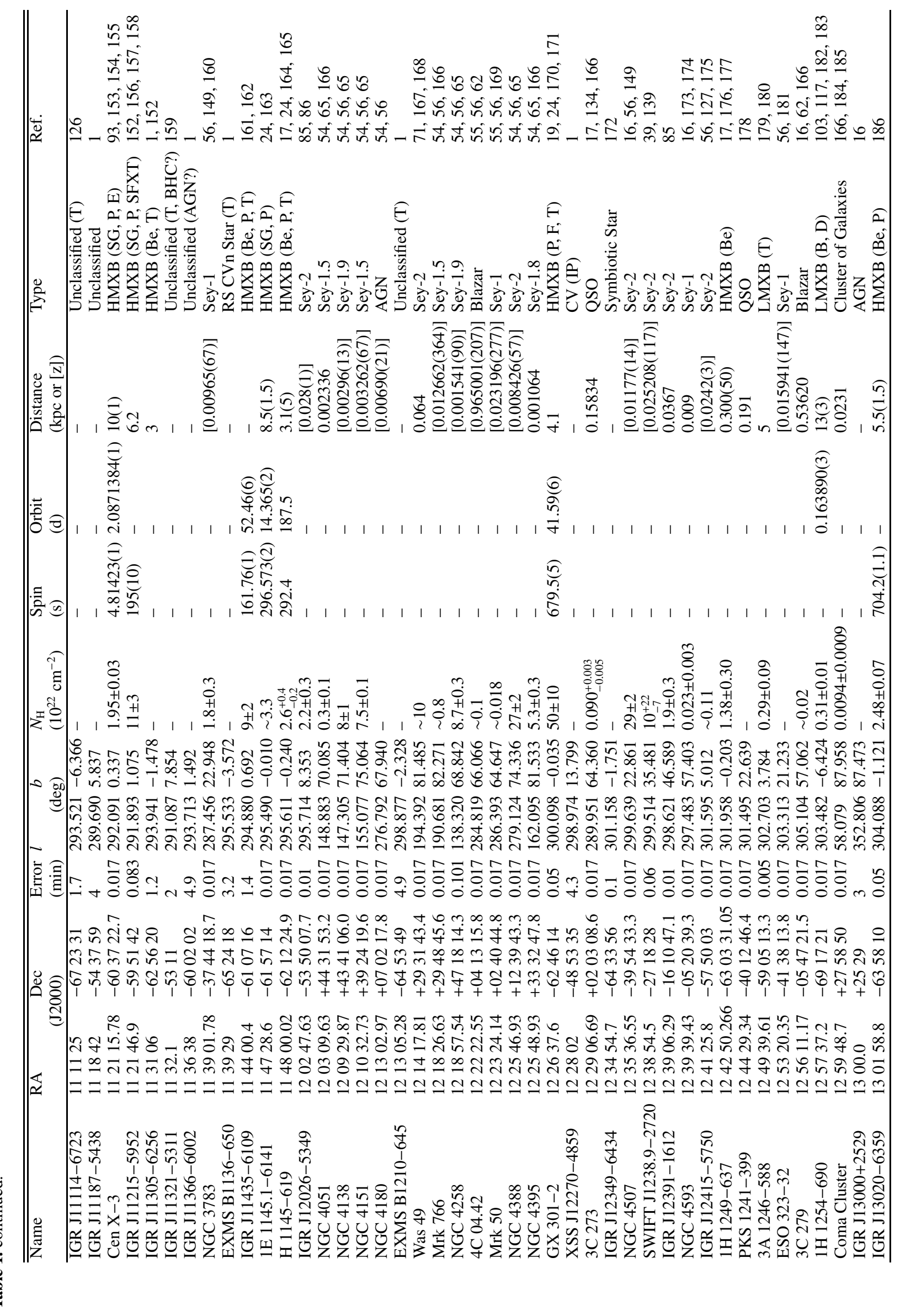




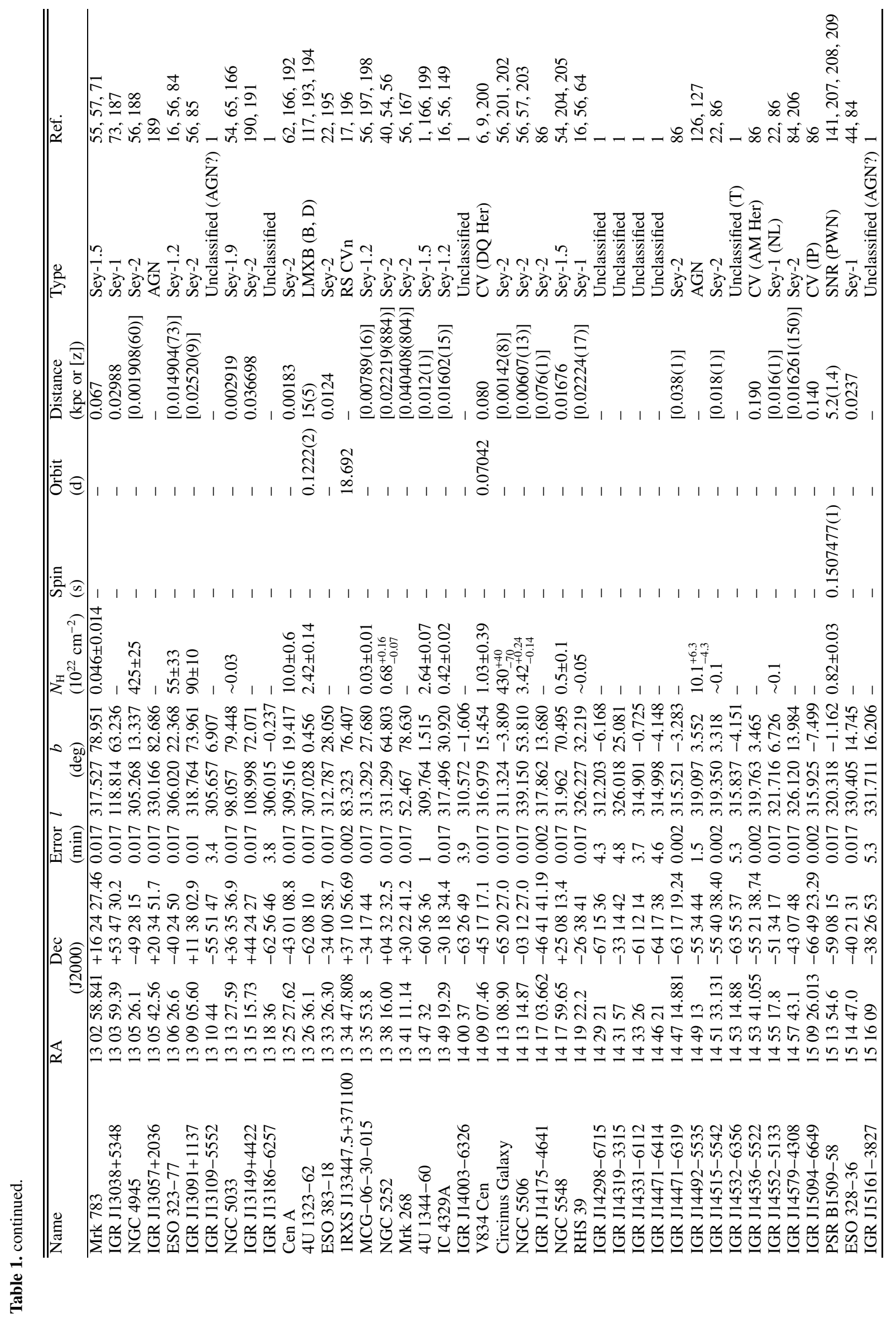




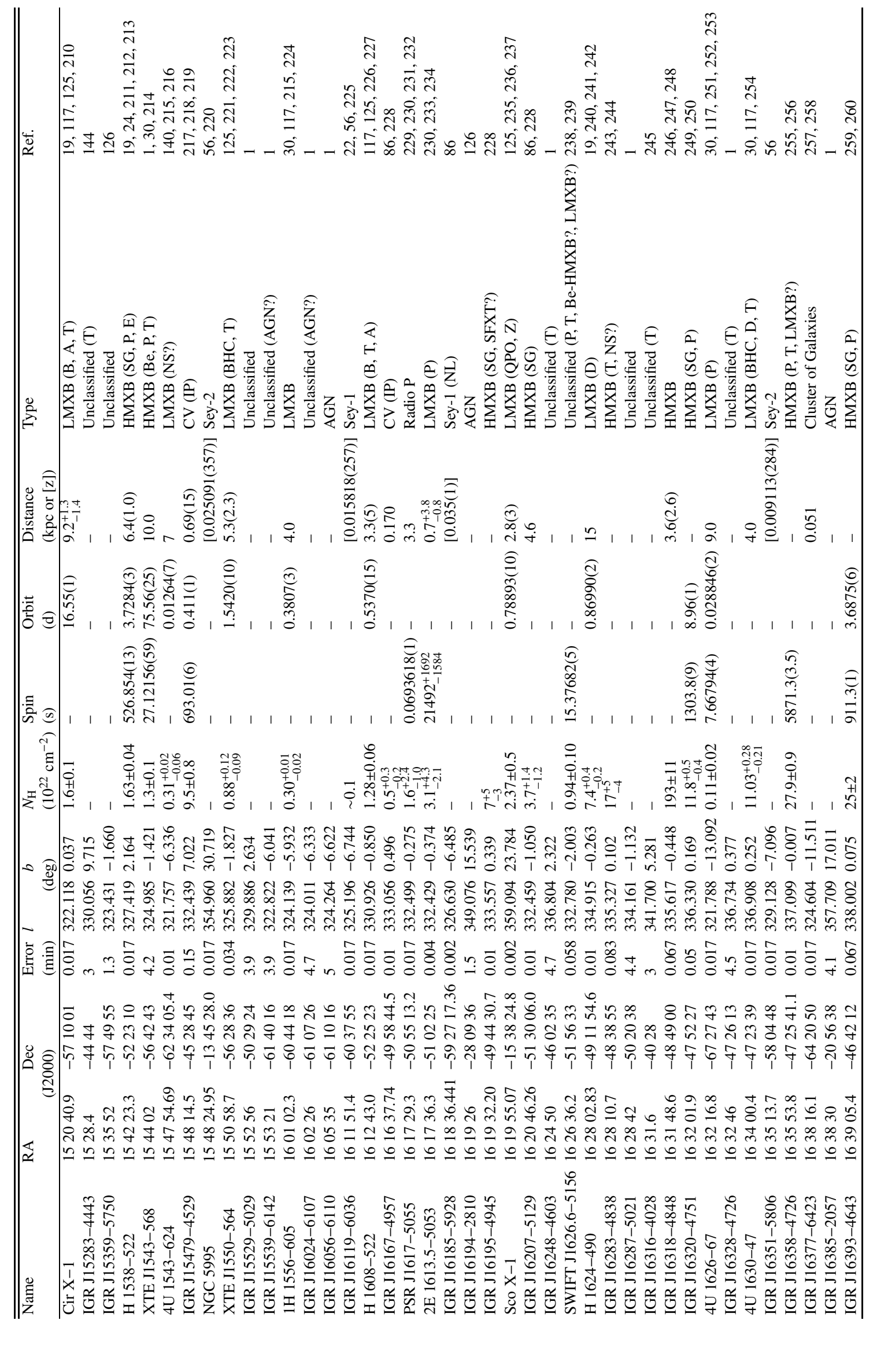




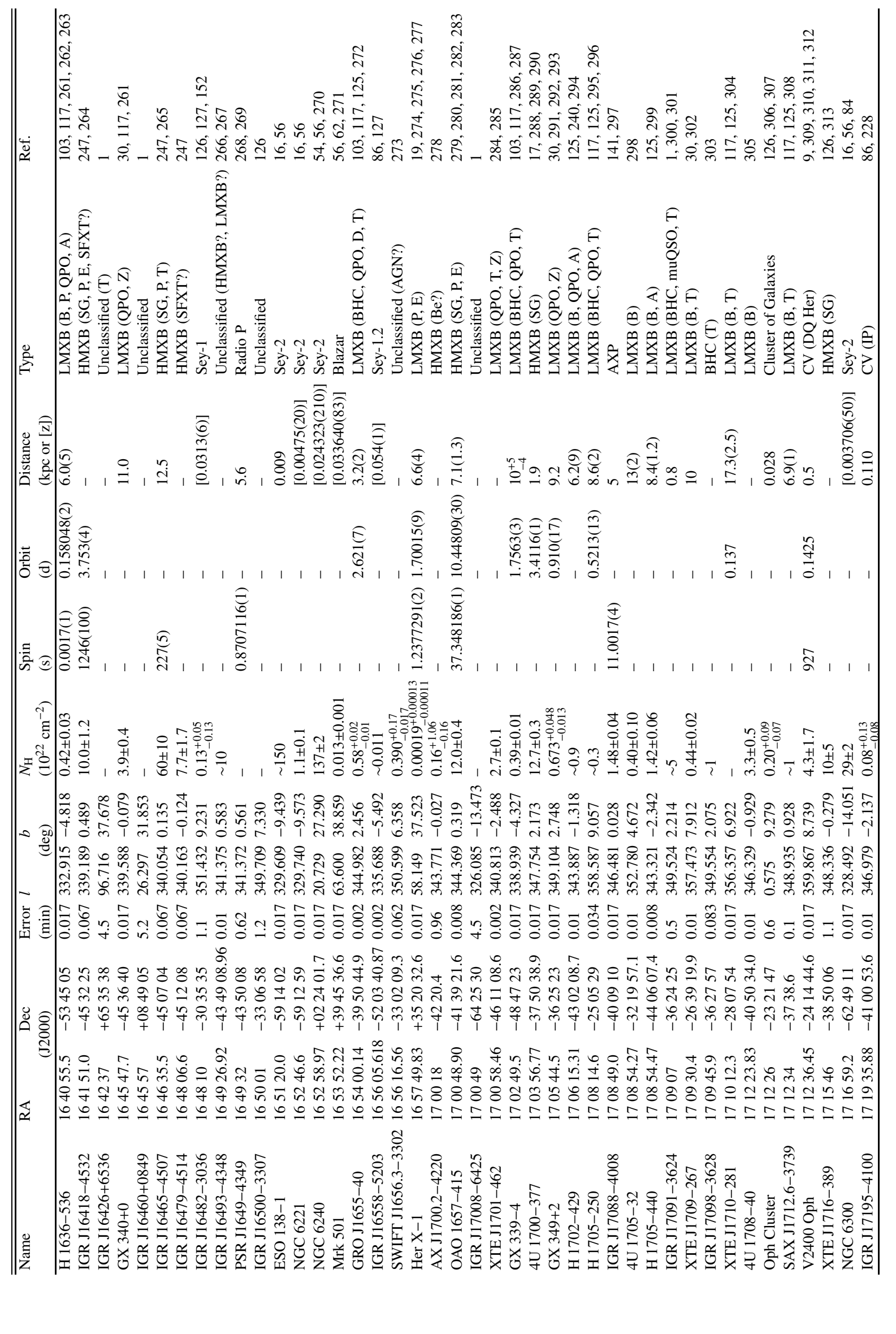




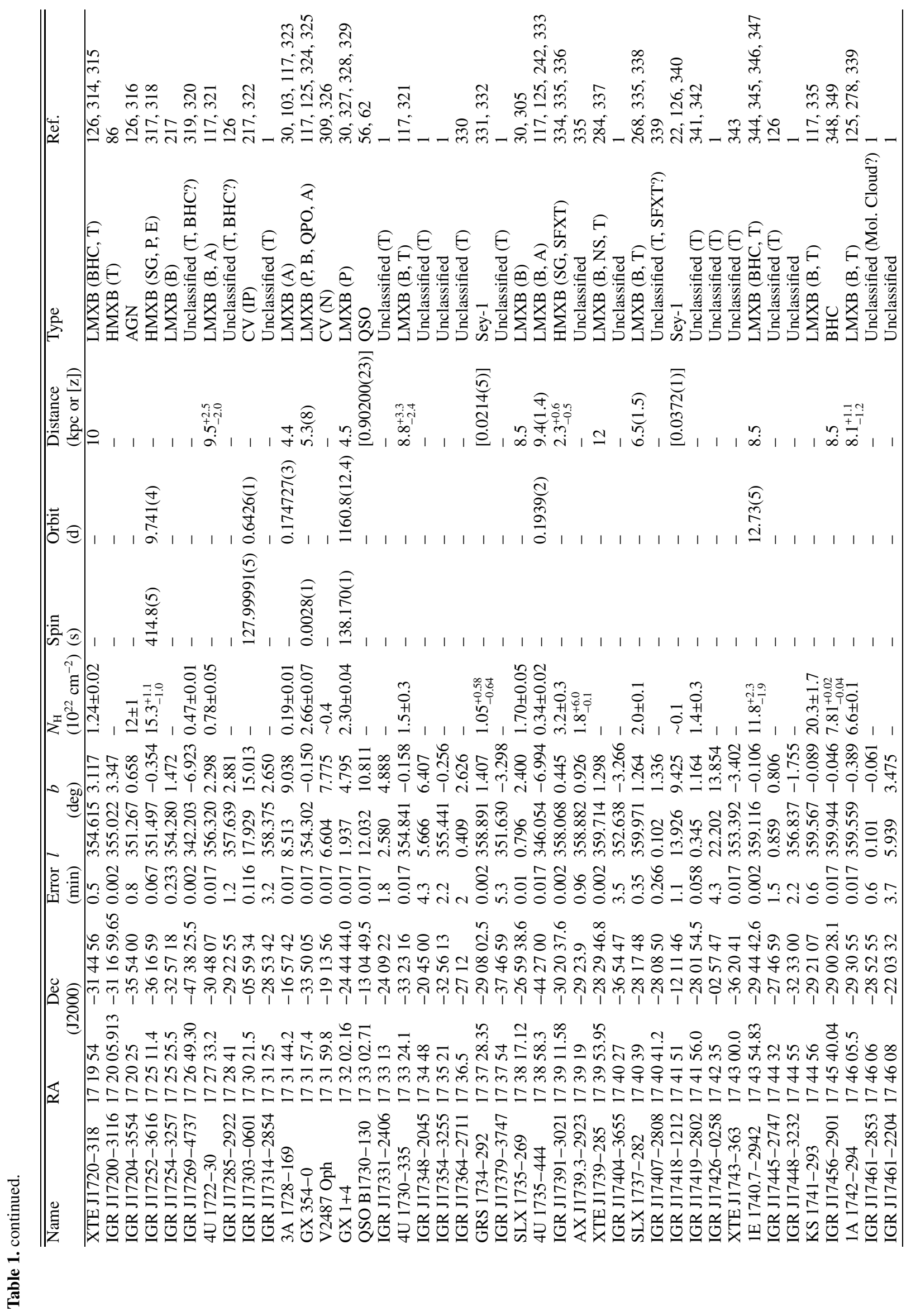




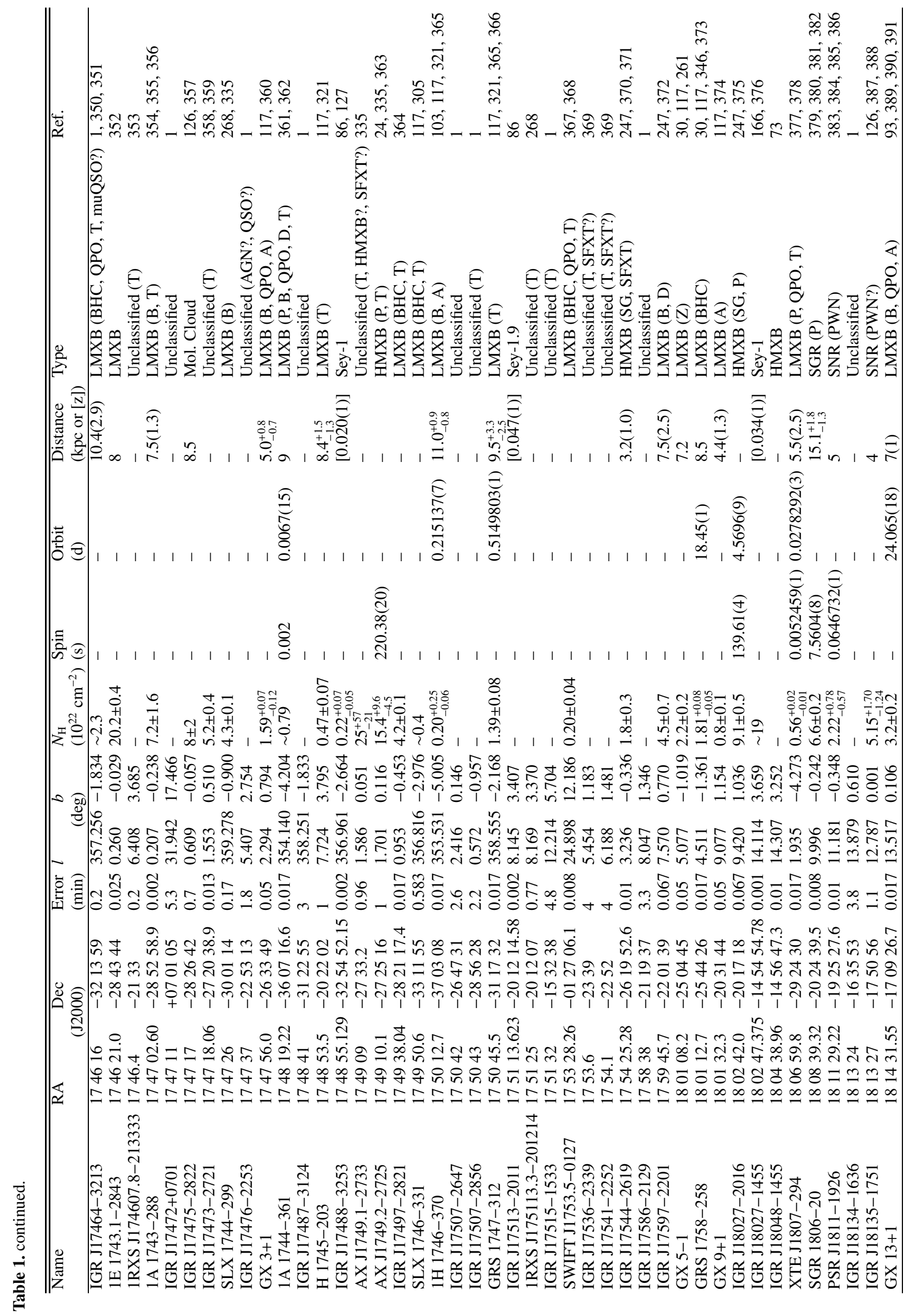


A. Bodaghee et al.: A description of INTEGRAL sources, Online Material p 11

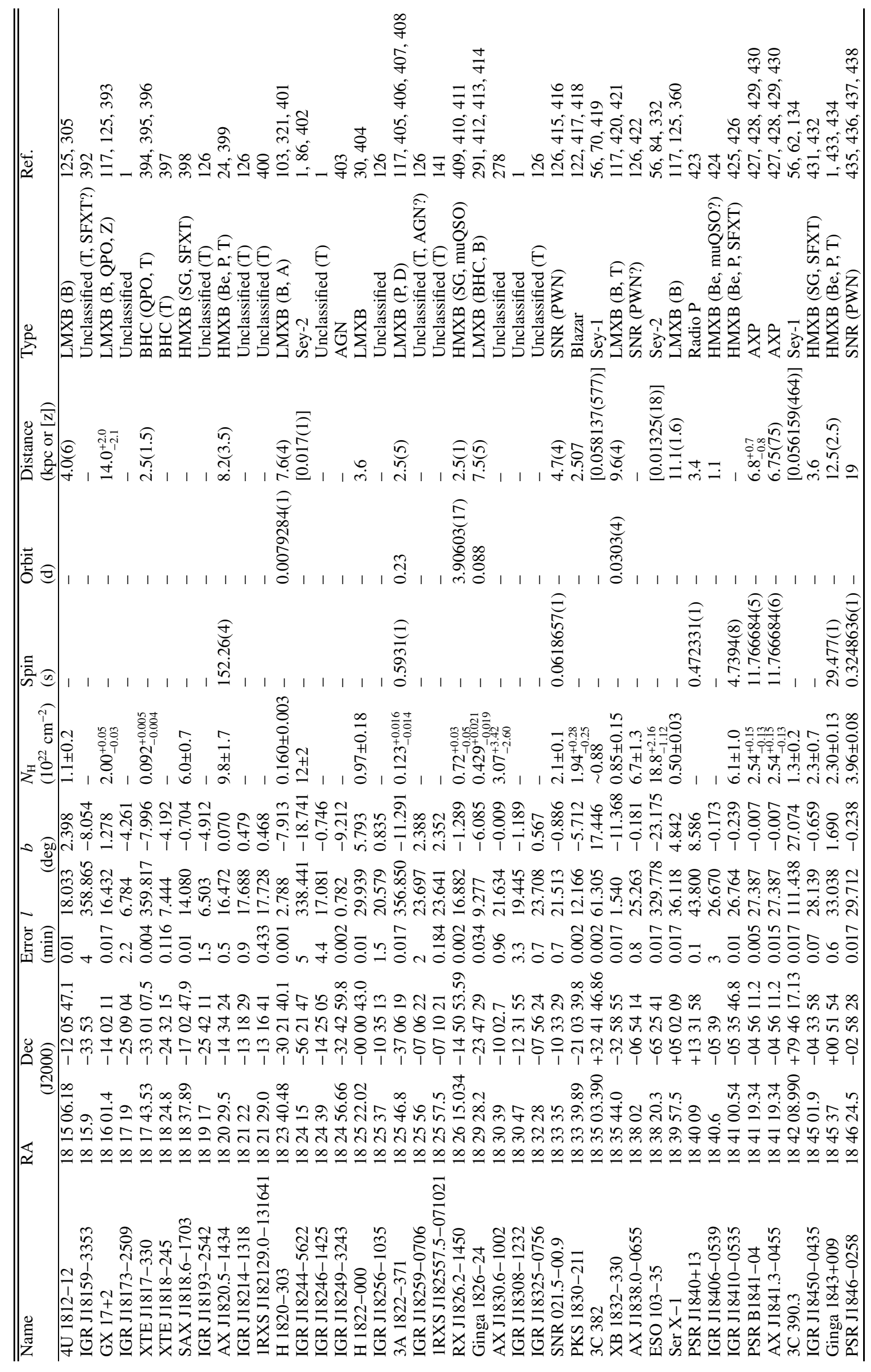




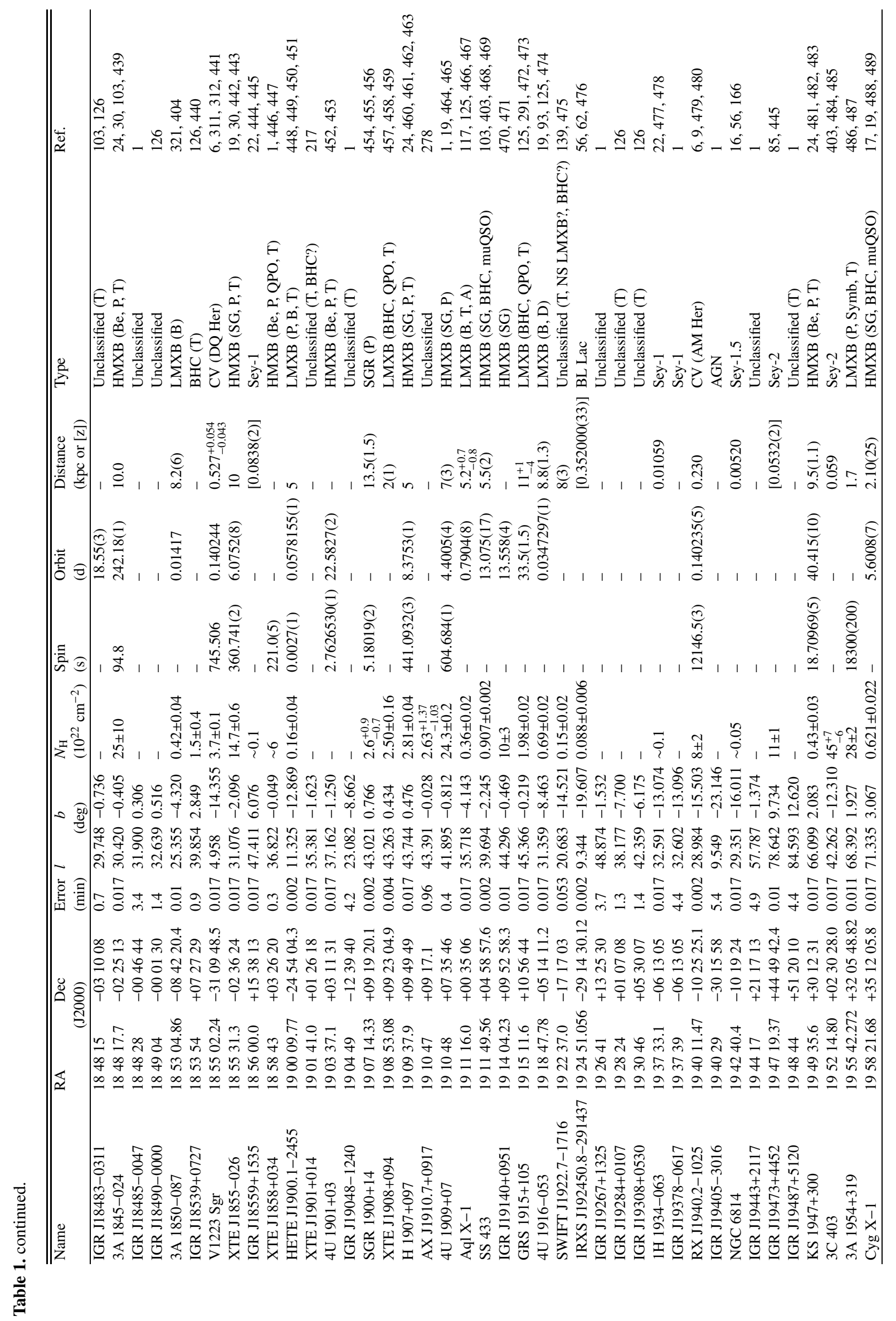


A. Bodaghee et al.: A description of INTEGRAL sources, Online Material p 13

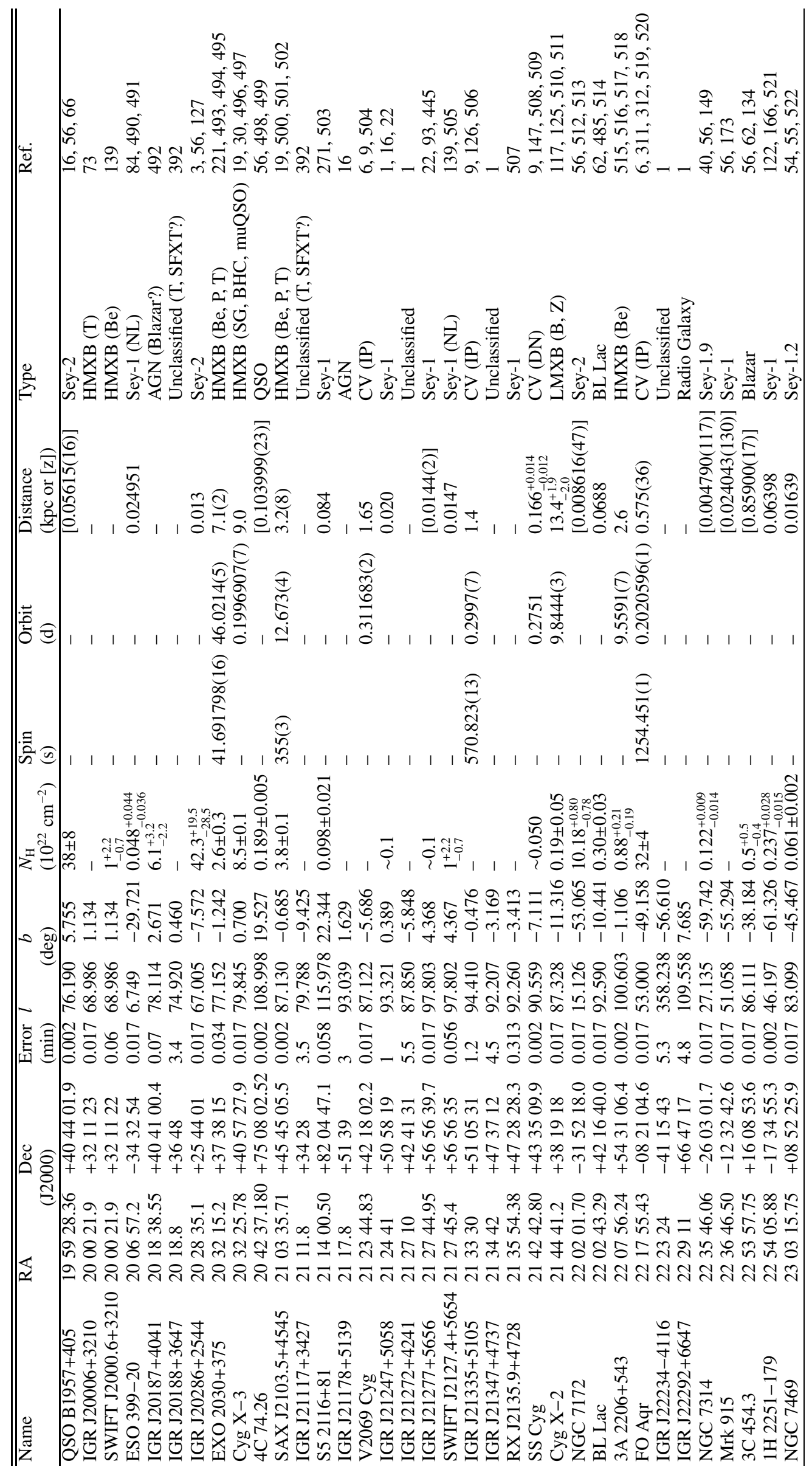


A. Bodaghee et al.: A description of INTEGRAL sources, Online Material p 14

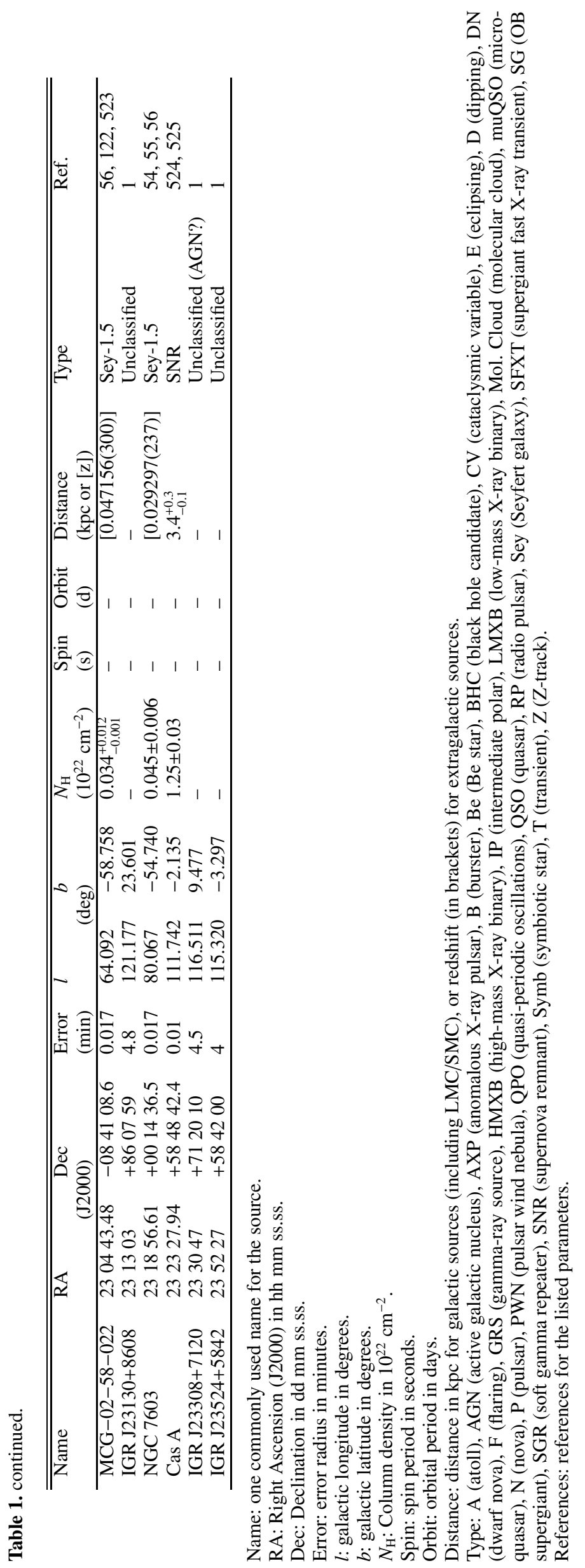


[1] Bird A.J., Malizia A., Bazzano A., et al., 2007, ApJ, in press, astro-ph/0611493

[2] Bikmaev I.F., Revnivtsev M.G., Burenin R.A., \& Sunyaev R.A., 2006, AstL, 32, 588

[3] Masetti N., Bassani L., Bazzano A., et al., 2006, A\&A, 455, 11

[4] Ruiz-Lapuente P., 2004, ApJ, 612, 357

[5] Kuiper L., Hartog P.R.D., \& Hermsen W., 2006, ATel, 939, 1

[6] Downes R., Webbink R.F., \& Shara M.M., 1997, PASP, 109, 345

[7] de Martino D., Matt G., Mukai K., et al., 2001, A\&A, 377, 499

[8] Bonnet-Bidaud J.M., Mouchet M., de Martino D., et al., 2001, A\&A, 374, 1003

[9] Barlow E.J., Knigge C., Bird A.J., et al., 2006, MNRAS, 372, 224

[10] Nowak M.A., Paizis A., Wilms J., et al., 2004, ATel, 369, 1

[11] Paizis A., Nowak M.A., Wilms J., et al., 2005, A\&A, 444, 357

[12] Burderi L., di Salvo T., Riggio A., et al., 2006, ChJAS, 6, 192

[13] Galloway D.K., Markwardt C.B., Morgan E.H., et al., 2005, ApJ, 622, L45

[14] Falanga M., Kuiper L., Poutanen J., et al., 2005, A\&A, 444, 15

[15] Laurent-Muehleisen S.A., Kollgaard R.I., Ryan P.J., et al., 1997, A\&AS, 122, 235

[16] Bassani L., Molina M., Malizia A., et al., 2006, ApJ, 636, L65

[17] Perryman M.A.C., Lindegren L., Kovalevsky J., et al., 1997, A\&A, 323, L49

[18] den Hartog P.R., Hermsen W., Kuiper L., et al., 2006, A\&A, 451, 587

[19] Wen L., Levine A.M., Corbet R.H.D., \& Bradt H.V., 2006, ApJS, 163, 372

[20] Negueruela I. \& Reig P., 2004, ATel, 285, 1

[21] Snellen I.A.G., McMahon R.G., Hook I.M., \& Browne I.W.A., 2002, MNRAS, 329, 700

[22] Sazonov S., Revnivtsev M., Krivonos R., et al., 2007, A\&A, 462, 57

[23] Yu P.-C. \& Hwang C.-Y., 2005, ApJ, 631, 720

[24] Liu Q.Z., van Paradijs J., \& van den Heuvel E.P.J., 2000, A\&AS, 147, 25

[25] Buckley D.A.H., Coe M.J., Stevens J.B., et al., 2001, MNRAS, 320, 281

[26] Corbet R., Marshall F.E., Lochner J.C., et al., 1998, IAU Circ., 6803, 1

[27] Galache J.L., Corbet R.H.D., Coe M.J., et al., 2005, ATel, 674,1

[28] Smith M.A., Cohen D.H., Gu M.F., et al., 2004, ApJ, 600, 972

[29] Harmanec P., Habuda P., Štefl S., et al., 2000, A\&A, 364, L85

[30] Grimm H.-J., Gilfanov M., \& Sunyaev R., 2002, A\&A, 391, 923

[31] Massey P., 2002, ApJS, 141, 81

[32] Naik S. \& Paul B., 2004, A\&A, 418, 655

[33] Corbet R.H.D., Finley J.P., \& Peele A.G., 1999, ApJ, 511, 876

[34] Crampton D., Hutchings J.B., \& Cowley A.P., 1985, ApJ, 299, 839

[35] Reig P., Chakrabarty D., Coe M.J., et al., 1996, A\&A, 311, 879
[36] Campana S., Gastaldello F., Stella L., et al., 2001, ApJ, 561, 924

[37] Tamura K., Tsunemi H., Kitamoto S., et al., 1992, ApJ, 389, 676

[38] Negueruela I. \& Okazaki A.T., 2001, A\&A, 369, 108

[39] da Costa L.N., Willmer C.N.A., Pellegrini P.S., et al., 1998, AJ, 116, 1

[40] Risaliti G., 2002, A\&A, 386, 379

[41] Polletta M., Bassani L., Malaguti G., et al., 1996, ApJS, 106, 399

[42] Reig P., Negueruela I., Papamastorakis G., et al., 2005, A\&A, 440, 637

[43] Loveday J., 1996, MNRAS, 278, 1025

[44] Hewitt A. \& Burbidge G., 1991, ApJS, 75, 297

[45] Hulleman F., van Kerkwijk M.H., \& Kulkarni S.R., 2004, A\&A, 416, 1037

[46] Göhler E., Wilms J., \& Staubert R., 2005, A\&A, 433, 1079

[47] Pigulski A., Kopacki G., \& Kołaczkowski Z., 2001, A\&A, 376, 144

[48] Mereghetti S., Tiengo A., Israel G.L., \& Stella L., 2000, A\&A, 354, 567

[49] Burenin R., Mescheryakov A., Sazonov S., et al., 2006, ATel, 883, 1

[50] Den Hartog P.R., Kuiper L., Hermsen W., et al., 2005, ATel, 394,1

[51] Kennea J.A., Racusin J.L., Burrows D.N., et al., 2005, ATel, 673, 1

[52] Argyle R.W. \& Eldridge P., 1990, MNRAS, 243, 504

[53] Kuiper L., Hermsen W., in 't Zand J., \& den Hartog P.R., 2005, ATel, 665, 1

[54] Cotton W.D., Condon J.J., \& Arbizzani E., 1999, ApJS, 125, 409

[55] Pfefferkorn F., Boller T., \& Rafanelli P., 2001, A\&A, 368, 797

[56] Paturel G. \& Petit C., 2002, LEDA (2002), 0

[57] Clements E.D., 1981, MNRAS, 197, 829

[58] Krongold Y., Nicastro F., Elvis M., et al., 2005, ApJ, 620, 165

[59] Arribas S., Mediavilla E., del Burgo C., \& García-Lorenzo B., 1999, ApJ, 511, 680

[60] Leahy D.A., Harrison F.A., \& Yoshida A., 1997, ApJ, 475, 823

[61] Taylor A.R. \& Gregory P.C., 1982, ApJ, 255, 210

[62] Ma C., Arias E.F., Eubanks T.M., et al., 1998, AJ, 116, 516

[63] Weaver K.A., Wilson A.S., Henkel C., \& Braatz J.A., 1999, ApJ, 520, 130

[64] Fischer J.-U., Hasinger G., Schwope A.D., et al., 1998, AN, 319,347

[65] Cappi M., Panessa F., Bassani L., et al., 2006, A\&A, 446, 459

[66] Titov O.A., 2004, ARep, 48, 941

[67] Karachentsev I.D., Karachentseva V.E., Kudrya Y.N., et al., 1999, Bull. Special Astrophys.Obs., 47, 5

[68] Keel W.C., 1996, ApJS, 106, 27

[69] Ishwara-Chandra C.H. \& Saikia D.J., 1999, MNRAS, 309, 100

[70] Beasley A.J., Gordon D., Peck A.B., et al., 2002, ApJS, 141, 13

[71] Enya K., Yoshii Y., Kobayashi Y., et al., 2002, ApJS, 141, 23

[72] Vrielmann S., Ness J.-U., \& Schmitt J.H.M.M., 2005, A\&A, 439, 287

[73] Burenin R., Mescheryakov A., Revnivtsev M., et al., 2006, ATel, 880, 1 
[74] Lawrence A., Rowan-Robinson M., Ellis R.S., et al., 1999, MNRAS, 308, 897

[75] Unger S.J., Norton A.J., Coe M.J., \& Lehto H.J., 1992, MNRAS, 256, 725

[76] Kreykenbohm I., Mowlavi N., Produit N., et al., 2005, A\&A, 433, L45

[77] Stella L., White N.E., Davelaar J., et al., 1985, ApJ, 288, L45

[78] Negueruela I., Roche P., Fabregat J., \& Coe M.J., 1999, MNRAS, 307, 695

[79] di Salvo T., Burderi L., Robba N.R., \& Guainazzi M., 1998, ApJ, 509, 897

[80] Delgado-Martí, H., Levine A.M., Pfahl E., \& Rappaport S.A., 2001, ApJ, 546, 455

[81] Lyubimkov L.S., Rostopchin S.I., Roche P., \& Tarasov A.E., 1997, MNRAS, 286, 549

[82] Fomalont E.B., Frey S., Paragi Z., et al., 2000, ApJS, 131, 95

[83] Motch C., Guillout P., Haberl F., et al., 1998, A\&AS, 132, 341

[84] Lauberts A., 1982, ESO/Uppsala survey of the ESO(B) atlas (Garching: European Southern Observatory (ESO), 1982)

[85] Sazonov S., Churazov E., Revnivtsev M., Vikhlinin A., \& Sunyaev R., 2005, A\&A, 444, L37

[86] Masetti N., Morelli L., Palazzi E., et al., 2006, A\&A, 459, 21

[87] Remillard R.A., Bradt H.V.D., Brissenden R.J.V., et al., 1993, AJ, 105, 2079

[88] Takata T., Yamada T., Saito M., Chamaraux P., \& Kazes I., 1994, A\&AS, 104, 529

[89] Kulkarni S.R., Kaplan D.L., Marshall H.L., et al., 2003, ApJ, 585, 948

[90] Haberl F., Dennerl K., \& Pietsch W., 2003, A\&A, 406, 471 [91] Dennerl K., Haberl F., \& Pietsch W., 1995, IAU Circ., 6184, 2

[92] Götz D., Mereghetti S., Merlini D., et al., 2006, A\&A, 448, 873

[93] Fuhrmeister B. \& Schmitt J.H.M.M., 2003, A\&A, 403, 247

[94] Naik S. \& Paul B., 2002, JApA, 23, 27

[95] Levine A.M., Rappaport S.A., \& Zojcheski G., 2000, ApJ, 541,194

[96] Han J.L. \& Tian W.W., 1999, A\&AS, 136, 571

[97] Kirsch M.G.F., Schönherr G., Kendziorra E., et al., 2006, A\&A, 453, 173

[98] Lyne A.G., Pritchard R.S., \& Graham-Smith F., 1993, MNRAS, 265, 1003

[99] Trimble V., 1973, PASP, 85, 579

[100] Mukherjee U. \& Paul B., 2005, A\&A, 431, 667

[101] Steele I.A., Negueruela I., Coe M.J., \& Roche P., 1998, MNRAS, 297, L5

[102] Cui W., Feng Y.X., Zhang S.N., et al., 2002, ApJ, 576, 357

[103] Levine A.M. \& Corbet R., 2006, ATel, 940, 1

[104] Haardt F., Galli M.R., Treves A., et al., 2001, ApJS, 133, 187

[105] Hirayama M., Nagase F., Endo T., et al., 2002, MNRAS, 333,603

[106] Livingstone M.A., Kaspi V.M., \& Gavriil F.P., 2005, ApJ, 633,1095

[107] Ramsay G. \& Cropper M., 2002, MNRAS, 334, 805

[108] Pavlenko E.P., 2006, Astrophysics, 49, 105

[109] Matt G., Bianchi S., de Rosa A., et al., 2006, A\&A, 445, 451

[110] Helou G. \& Walker D.W., eds. 1988, IRAS catalogs and atlases. Volume 7: The small scale structure catalog
[111] Kennea J.A., Markwardt C.B., Tueller J., et al., 2005, ATel, 677,1

[112] Morelli L., Masetti N., Bassani L., et al., 2006, ATel, 785, 1

[113] Chenevez J., Budtz-Jorgensen C., Lund N., et al., 2004, ATel, 223, 1

[114] Mauch T., Murphy T., Buttery H.J., et al., 2003, MNRAS, 342,1117

[115] Brinkmann W. \& Siebert J., 1994, A\&A, 285, 812

[116] Cappi M., Bassani L., Comastri A., et al., 1999, A\&A, 344,857

[117] Liu Q.Z., van Paradijs J., \& van den Heuvel E.P.J., 2001, A\&A, 368, 1021

[118] Piraino S., Santangelo A., Ford E.C., \& Kaaret P., 1999, A\&A, 349, L77

[119] Ford E., Kaaret P., Tavani M., et al., 1997, ApJ, 475, L123 [120] Paerels F., Brinkman A.C., van der Meer R.L.J., et al., 2001, ApJ, 546, 338

[121] Araujo-Betancor S., Gänsicke B.T., Hagen H.-J., et al., 2003, A\&A, 406, 213

[122] Barkhouse W.A. \& Hall P.B., 2001, AJ, 121, 2843

[123] Malizia A., Bassani L., Capalbi M., et al., 2003, A\&A, 406,105

[124] Sidoli L., Parmar A.N., \& Oosterbroek T., 2005, A\&A, 429, 291

[125] Jonker P.G. \& Nelemans G., 2004, MNRAS, 354, 355

[126] Bird A.J., Barlow E.J., Bassani L., et al., 2006, ApJ, 636, 765

[127] Landi R., Malizia A., Bassani L., et al., 2006, astroph/0610358

[128] Revnivtsev M.G., Sazonov S.Y., Molkov S.V., et al., 2006, AstL, 32, 145

[129] Porquet D., Reeves J.N., O’Brien P., \& Brinkmann W., 2004, A\&A, 422, 85

[130] Pavlov G.G., Zavlin V.E., Sanwal D., et al., 2001, ApJ, 552, L129

[131] Caraveo P.A., De Luca A., Mignani R.P., \& Bignami G.F., 2001, ApJ, 561, 930

[132] Belloni T., Hasinger G., Pietsch W., et al., 1993, A\&A, 271, 487

[133] Kennea J.A. \& Campana S., 2006, ATel, 818, 1

[134] Grandi P., Malaguti G., \& Fiocchi M., 2006, ApJ, 642, 113

[135] Orlandini M., dal Fiume D., Frontera F., et al., 1998, A\&A, 332,121

[136] Kreykenbohm I., Coburn W., Wilms J., et al., 2002, A\&A, 395,129

[137] Sadakane K., Hirata R., Jugaku J., et al., 1985, ApJ, 288, 284

[138] Kirhakos S., Strauss M.A., Yahil A., et al., 1991, AJ, 102, 1933

[139] Tueller J., Barthelmy S., Burrows D., et al., 2005, ATel, 669,1

[140] Juett A.M. \& Chakrabarty D., 2003, ApJ, 599, 498

[141] Voges W., Aschenbach B., Boller T., et al., 1999, A\&A, 349,389

[142] Pineda F. \& Schnopper H.W., 1978, IAU Circ., 3190, 3

[143] Balestra I., Bianchi S., \& Matt G., 2004, A\&A, 415, 437

[144] Paizis A., Gotz D., Sidoli L., et al., 2006, ATel, 865, 1

[145] Shrader C.R., Sutaria F.K., Singh K.P., \& Macomb D.J., 1999, ApJ, 512, 920

[146] Ajello M., Greiner J., Küpcü Yoldas A., et al., 2006, ATel, 864,1

[147] Zacharias N. Urban S.E. Zacharias M.I. et al., 2003, VizieR Online Data Catalog, 1289 
[148] Ho L.C., Filippenko A.V., \& Sargent W.L., 1995, ApJS, 98,477

[149] Veron-Cetty M.-P. \& Veron P., 1996, A Catalogue of quasars and active nuclei (ESO Scientific Report, Garching: European Southern Observatory (ESO), 1996, 7th ed.)

[150] Vignali C. \& Comastri A., 2002, A\&A, 381, 834

[151] Reig P. \& Roche P., 1999, MNRAS, 306, 100

[152] Masetti N., Pretorius M.L., Palazzi E., et al., 2006, A\&A, 449,1139

[153] Burderi L., Di Salvo T., Robba N.R., et al., 2000, ApJ, 530, 429

[154] Nagase F., Corbet R.H.D., Day C.S.R., et al., 1992, ApJ, 396, 147

[155] Hutchings J.B., Cowley A.P., Crampton D., et al., 1979, ApJ, 229, 1079

[156] Steeghs D., Torres M.A.P., \& Jonker P.G., 2006, ATel, 768, 1

[157] Smith D.M., Bezayiff N., \& Negueruela I., 2006, ATel, 773,1

[158] Sidoli L., Paizis A., \& Mereghetti S., 2006, A\&A, 450, L9 [159] Krivonos R., Molkov S., Revnivtsev M., et al., 2005, ATel, 545,1

[160] De Rosa A., Piro L., Fiore F., et al., 2002, A\&A, 387, 838

[161] in 't Zand J. \& Heise J., 2004, ATel, 362, 1

[162] Corbet R.H.D. \& Remillard R., 2005, ATel, 377, 1

[163] Ray P.S. \& Chakrabarty D., 2002, ApJ, 581, 1293

[164] Cook M.C. \& Warwick R.S., 1987, MNRAS, 227, 661

[165] Stevens J.B., Reig P., Coe M.J., et al., 1997, MNRAS, 288, 988

[166] Beckmann V., Gehrels N., Shrader C.R., \& Soldi S., 2006, ApJ, 638, 642

[167] Becker R.H., White R.L., \& Helfand D.J., 1995, ApJ, 450, 559

[168] Risaliti G., Gilli R., Maiolino R., \& Salvati M., 2000, A\&A, 357, 13

[169] Li J. \& Jin W., 1996, A\&AS, 120, 201

[170] La Barbera A., Segreto A., Santangelo A., et al., 2005, A\&A, 438, 617

[171] Leahy D.A., 2002, A\&A, 391, 219

[172] Tueller J., Gehrels N., Mushotzky R.F., et al., 2005, ATel, 591,1

[173] Clements E.D., 1983, MNRAS, 204, 811

[174] Guainazzi M., Perola G.C., Matt G., et al., 1999, A\&A, 346, 407

[175] Fairall A.P., Woudt P.A., \& Kraan-Korteweg R.C., 1998, A\&AS, 127, 463

[176] Torrejón J.M. \& Orr A., 2001, A\&A, 377, 148

[177] Perryman M.A.C. \& ESA., 1997, The HIPPARCOS and TYCHO catalogues. Astrometric and photometric star catalogues derived from the ESA HIPPARCOS Space Astrometry Mission (Publisher: Noordwijk, Netherlands: ESA Publications Division, 1997, Series: ESA SP Series vol no: 1200, ISBN: 9290923997 (set))

[178] Jackson C.A., Wall J.V., Shaver P.A., et al., 2002, A\&A, 386, 97

[179] Bassa C.G., Jonker P.G., in 't Zand J.J.M., \& Verbunt F., 2006, A\&A, 446, L17

[180] Boller T., Haberl F., Voges W., Piro L., \& Heise J., 1997, IAU Circ., 6546, 1

[181] Jerjen H. \& Dressler A., 1997, A\&AS, 124, 1

[182] Boirin L. \& Parmar A.N., 2003, A\&A, 407, 1079

[183] in 't Zand J.J.M., Kuulkers E., Verbunt F., et al., 2003 ,

A\&A, 411, L487

[184] Mahdavi A. \& Geller M.J., 2001, ApJ, 554, L129
[185] Arnaud M., Aghanim N., Gastaud R., et al., 2001, A\&A, 365, L67

[186] Chernyakova M., Lutovinov A., Rodriguez J., \& Revnivtsev M., 2005, MNRAS, 364, 455

[187] Bicay M.D., Stepanian J.A., Chavushyan V.H., et al., 2000, A\&AS, 147, 169

[188] Done C., Madejski G.M., Życki P.T., \& Greenhill L.J., 2003, ApJ, 588, 763

[189] Odewahn S.C. \& Aldering G., 1996, Private Communication, 1

[190] Palumbo G.G.C., et al., 1988, in Accurate positions of Zwicky galaxies (1988), 0-+

[191] Nilson P., 1973, Nova Acta Regiae Soc.Sci.Upsaliensis Ser.V, 0

[192] Evans D.A., Kraft R.P., Worrall D.M., et al., 2004, ApJ, 612,786

[193] Church M.J., Reed D., Dotani T., Bałucińska-Church M., \& Smale A.P., 2005, MNRAS, 359, 1336

[194] Parmar A.N., Gottwald M., van der Klis M., \& van Paradijs J., 1989, ApJ, 338, 1024

[195] Kaldare R., Colless M., Raychaudhury S., \& Peterson B.A., 2003, MNRAS, 339, 652

[196] Demircan O., Eker Z., Karataş Y., \& Bilir S., 2006, MNRAS, 366, 1511

[197] Willmer C.N.A., Maia M.A.G., Mendes S.O., et al., 1999, AJ, 118, 1131

[198] Papadakis I.E., Kazanas D., \& Akylas A., 2005, ApJ, 631, 727

[199] Piconcelli E., Sánchez-Portal M., Guainazzi M., et al., 2006, A\&A, 453, 839

[200] Imamura J.N., Steiman-Cameron T.Y., \& Wolff M.T., 2000, PASP, 112, 18

[201] Wright A.E., Griffith M.R., Hunt A.J., et al., 1996, ApJS, 103,145

[202] Matt G., Guainazzi M., Maiolino R., et al., 1999, A\&A, 341, L39

[203] Bianchi S., Balestra I., Matt G., et al., 2003, A\&A, 402, 141

[204] Nicastro F., Piro L., De Rosa A., et al., 2000, ApJ, 536, 718

[205] Crenshaw D.M. \& Kraemer S.B., 1999, ApJ, 521, 572

[206] West R.M., Surdej J., Schuster H.-E., et al., 1981, A\&AS, 46,57

[207] DeLaney T., Gaensler B.M., Arons J., \& Pivovaroff M.J., 2006, ApJ, 640, 929

[208] Livingstone M.A., Kaspi V.M., Gavriil F.P., \& Manchester R.N., 2005, ApJ, 619, 1046

[209] Gaensler B.M., Brazier K.T.S., Manchester R.N., et al., 1999, MNRAS, 305, 724

[210] Iaria R., Di Salvo T., Burderi L., \& Robba N.R., 2001, ApJ, 561, 321

[211] Robba N.R., Burderi L., Di Salvo T., et al., 2001, ApJ, 562, 950

[212] Baykal A., Inam S.Ç., \& Beklen E., 2006, A\&A, 453, 1037 [213] Reynolds A.P., Bell S.A., \& Hilditch R.W., 1992, MNRAS, 256, 631

[214] in 't Zand J.J.M., Corbet R.H.D., \& Marshall F.E., 2001, ApJ, 553, L165

[215] Farinelli R., Frontera F., Masetti N., et al., 2003, A\&A, 402, 1021

[216] Wang Z. \& Chakrabarty D., 2004, ApJ, 616, L139

[217] Stephen J.B., Bassani L., Molina M., et al., 2005, A\&A, 432, L49 
[218] Haberl F., Motch C., \& Zickgraf F.-J., 2002, A\&A, 387, 201

[219] de Martino D., Bonnet-Bidaud J.-M., Mouchet M., et al., 2006, A\&A, 449, 1151

[220] Klemola A.R., Jones B.F., \& Hanson R.B., 1987, AJ, 94, 501

[221] Kazarovets E.V., Samus N.N., \& Durlevich O.V., 2001, IBVS, 5135, 1

[222] Corbel S., Tomsick J.A., \& Kaaret P., 2006, ApJ, 636, 971

[223] Orosz J.A., Groot P.J., van der Klis M., et al., 2002, ApJ, 568,845

[224] Smale A.P., 1991, PASP, 103, 636

[225] Woudt P.A. \& Kraan-Korteweg R.C., 2001, A\&A, 380, 441

[226] Greco M.V., Miller J.M., \& Steeghs D., 2006, ATel, 858, 1 [227] Wachter S., Hoard D.W., Bailyn C.D., et al., 2002, ApJ, 568, 901

[228] Tomsick J.A., Chaty S., Rodriguez J., et al., 2006, ApJ, 647, 1309

[229] Kaspi V.M., Crawford F., Manchester R.N., et al., 1998, ApJ, 503, L161

[230] Gotthelf E.V., Petre R., \& Hwang U., 1997, ApJ, 487, L175

[231] Torii K., Gotthelf E.V., Vasisht G., et al., 2000, ApJ, 534, L71

[232] Caswell J.L., Murray J.D., Roger R.S., et al., 1975, A\&A, 45, 239

[233] Garmire G.P., Pavlov G.G., Garmire A.B., \& Zavlin V.E., 2000, IAU Circ., 7350, 2

[234] Reynoso E.M., Green A.J., Johnston S., et al., 2004, PASA, 21, 82

[235] McNamara B.J., Harrison T.E., Zavala R.T., et al., 2003, AJ, 125, 1437

[236] Bradshaw C.F., Geldzahler B.J., \& Fomalont E.B., 2003, ApJ, 592, 486

[237] Vanderlinde K.W., Levine A.M., \& Rappaport S.A., 2003, PASP, 115, 739

[238] Campana S., Belloni T., Homan J., et al., 2006, ATel, 688, 1

[239] Markwardt C.B. \& Swank J.H., 2005, ATel, 679, 1

[240] Wachter S., Wellhouse J.W., Patel S.K., et al., 2005, ApJ, 621,393

[241] Parmar A.N., Oosterbroek T., Boirin L., \& Lumb D., 2002, A\&A, 386, 910

[242] Christian D.J. \& Swank J.H., 1997, ApJS, 109, 177

[243] Kennea J.A., Burrows D.N., Nousek J.A., et al., 2005, ATel, 459, 1

[244] Beckmann V., Kennea J.A., Markwardt C., et al., 2005, ApJ, 631, 506

[245] Rodriguez J. \& Goldwurm A., 2003, ATel, 201, 1

[246] Schartel N., Ehle M., Breitfellner M., et al., 2003, IAU Circ., 8072, 3

[247] Walter R., Zurita Heras J.A., Bassani L., et al., 2006, A\&A, 453, 133

[248] Filliatre P. \& Chaty S., 2004, ApJ, 616, 469

[249] Rodriguez J., Bodaghee A., Kaaret P., et al., 2006, MNRAS, 366, 274

[250] Corbet R., Barbier L., Barthelmy S., et al., 2005, ATel, 649,1

[251] Orlandini M., Fiume D.D., Frontera F., et al., 1998, ApJ, 500, L163

[252] Owens A., Oosterbroek T., \& Parmar A.N., 1997, A\&A, 324, L9
[253] Middleditch J., Mason K.O., Nelson J.E., \& White N.E., 1981, ApJ, 244, 1001

[254] Tomsick J.A., Corbel S., Goldwurm A., \& Kaaret P., 2005, ApJ, 630, 413

[255] Kouveliotou C., Patel S., Tennant A., et al., 2003, IAU Circ., 8109, 2

[256] Patel S.K., Zurita Heras J.A., Del Santo M., et al., 2006, ApJ, in press, astro-ph/0610768

[257] Ebeling H., Mullis C.R., \& Tully R.B., 2002, ApJ, 580, 774

[258] McHardy I.M., Lawrence A., Pye J.P., \& Pounds K.A., 1981, MNRAS, 197, 893

[259] Bodaghee A., Walter R., Zurita Heras J.A., et al., 2006, A\&A, 447, 1027

[260] Thompson T.W.J., Tomsick J.A., Rothschild R.E., et al., 2006, ApJ, 649, 373

[261] Vrtilek S.D., McClintock J.E., Seward F.D., et al., 1991, ApJS, 76, 1127

[262] Strohmayer T.E. \& Markwardt C.B., 2002, ApJ, 577, 337

[263] Galloway D.K., Psaltis D., Muno M.P., \& Chakrabarty D., 2006, ApJ, 639, 1033

[264] Corbet R., Barbier L., Barthelmy S., et al., 2006, ATel, 779,1

[265] Smith D.M., 2004, ATel, 338, 1

[266] Kuiper L., Jonker P., Hermsen W., \& O’Brien K., 2005, ATel, 654, 1

[267] Markwardt C.B., Swank J.H., \& Smith E., 2005, ATel, 465, 1

[268] Ebisawa K., Bourban G., Bodaghee A., et al., 2003, A\&A, 411, L59

[269] Manchester R.N., Lyne A.G., Camilo F., et al., 2001, MNRAS, 328, 17

[270] Netzer H., Lemze D., Kaspi S., et al., 2005, ApJ, 629, 739

[271] Donato D., Sambruna R.M., \& Gliozzi M., 2005, A\&A, 433,1163

[272] Brocksopp C., McGowan K.E., Krimm H., et al., 2006, MNRAS, 365, 1203

[273] Tueller J., Markwardt C., Ajello M., et al., 2006, ATel, 835,1

[274] Kinnunen T. \& Skiff B.A., 2000, IBVS, 4863, 1

[275] Oosterbroek T., Parmar A.N., Dal Fiume D., et al., 2000, A\&A, 353, 575

[276] Kuster M., Wilms J., Staubert R., et al., 2005, A\&A, 443, 753

[277] Reynolds A.P., Quaintrell H., Still M.D., et al., 1997, MNRAS, 288, 43

[278] Sugizaki M., Mitsuda K., Kaneda H., et al., 2001, ApJS, 134, 77

[279] Chakrabarty D., Wang Z., Juett A.M., et al., 2002, ApJ, 573, 789

[280] Orlandini M., dal Fiume D., del Sordo S., et al., 1999, A\&A, 349, L9

[281] Baykal A., 2000, MNRAS, 313, 637

[282] Chakrabarty D., Grunsfeld J.M., Prince T.A., et al., 1993, ApJ, 403, L33

[283] Audley M.D., Nagase F., Mitsuda K., et al., 2006, MNRAS, 367, 1147

[284] Krauss M.I., Juett A.M., Chakrabarty D., et al., 2006, ATel, 777, 1

[285] Kennea J.A., Marshall F.E., Steeghs D., et al., 2006, ATel, 704, 1

[286] Miller J.M., Raymond J., Fabian A.C., et al., 2004, ApJ, 601,450 
A. Bodaghee et al.: A description of INTEGRAL sources, Online Material p 19

[287] Hynes R.I., Steeghs D., Casares J., et al., 2004, ApJ, 609, 317

[288] Boroson B., Vrtilek S.D., Kallman T., \& Corcoran M., 2003, ApJ, 592, 516

[289] Hong J. \& Hailey C.J., 2004, ApJ, 600, 743

[290] Ankay A., Kaper L., de Bruijne J.H.J., et al., 2001, A\&A, 370,170

[291] Kazarovets E.V., Samus N.N., \& Durlevich O.V., 2000, IBVS, 4870, 1

[292] Iaria R., Di Salvo T., Robba N.R., et al., 2004, ApJ, 600, 358

[293] Wachter S. \& Margon B., 1996, AJ, 112, 2684

[294] Oosterbroek T., Penninx W., van der Klis M., et al., 1991, A\&A, 250, 389

[295] Asai K., Dotani T., Hoshi R., et al., 1998, PASJ, 50, 611

[296] Remillard R.A., Orosz J.A., McClintock J.E., \& Bailyn C.D., 1996, ApJ, 459, 226

[297] Rea N., Oosterbroek T., Zane S., et al., 2005, MNRAS, 361,710

[298] in 't Zand J.J.M., Cornelisse R., \& Méndez M., 2005, A\&A, 440, 287

[299] Di Salvo T., Iaria R., Méndez M., et al., 2005, ApJ, 623, L121

[300] in 't Zand J.J.M., Heise J., Lowes P., \& Ubertini P., 2003, ATel, 160, 1

[301] Negueruela I. \& Schurch M., 2007, A\&A, 461, 631

[302] Jonker P.G., Méndez M., Nelemans G., et al., 2003, MNRAS, 341, 823

[303] Kennea J.A., Burrows D.N., Nousek J.A., et al., 2005, ATel, 476, 1

[304] Ritter H. \& Kolb U., 2003, A\&A, 404, 301

[305] Wilson C.A., Patel S.K., Kouveliotou C., et al., 2003, ApJ, 596,1220

[306] Edge A.C. \& Stewart G.C., 1991, MNRAS, 252, 414

[307] Nevalainen J., Oosterbroek T., Bonamente M., \& Colafrancesco S., 2004, ApJ, 608, 166

[308] Cocchi M., Bazzano A., Natalucci L., et al., 2001, Mem. Soc. Astron. Italiana, 72, 757

[309] Downes R.A., Webbink R.F., Shara M.M., et al., 2001, PASP, 113, 764

[310] de Martino D., Matt G., Belloni T., et al., 2004, A\&A, 415, 1009

[311] Norton A.J., Wynn G.A., \& Somerscales R.V., 2004, ApJ, 614, 349

[312] Suleimanov V., Revnivtsev M., \& Ritter H., 2005, A\&A, 435,191

[313] Cornelisse R., Charles P.A., \& Robertson C., 2006, MNRAS, 366, 918

[314] Cadolle Bel M., Rodriguez J., Sizun P., et al., 2004, A\&A, 426, 659

[315] Nagata T., Kato D., Baba D., et al., 2003, PASJ, 55, L73

[316] Bykov A.M., Krassilchtchikov A.M., Uvarov Y.A., et al., 2006, A\&A, 449, 917

[317] Zurita Heras J.A., de Cesare G., Walter R., et al., 2006, A\&A, 448, 261

[318] Corbet R.H.D., Markwardt C.B., \& Swank J.H., 2005, ApJ, 633, 377

[319] Steeghs D., Torres M.A.P., Koviak K., et al., 2005, ATel, 629,1

[320] Kennea J.A., Burrows D.N., Chester M., et al., 2005, ATel, 632,1

[321] Kuulkers E., den Hartog P.R., in 't Zand J.J.M., et al., 2003, A\&A, 399, 663
[322] Gänsicke B.T., Marsh T.R., Edge A., et al., 2005, MNRAS, 361,141

[323] Church M.J. \& Balucińska-Church M., 2001, A\&A, 369, 915

[324] D’ Aí, A., di Salvo T., Iaria R., et al., 2006, A\&A, 448, 817 [325] Strohmayer T.E., Zhang W., Swank J.H., et al., 1996, ApJ, 469, L9

[326] Hernanz M. \& Sala G., 2002, Science, 298, 393

[327] Munari U. \& Zwitter T., 2002, A\&A, 383, 188

[328] Paul B., Dotani T., Nagase F., et al., 2005, ApJ, 627, 915

[329] Hinkle K.H., Fekel F.C., Joyce R.R., et al., 2006, ApJ, 641, 479

[330] Chelovekov I.V., Grebenev S.A., \& Sunyaev R.A., 2006, AstL, 32, 456

[331] Marti J., Mirabel I.F., Chaty S., \& Rodriguez L.F., 1998, A\&A, 330, 72

[332] Molina M., Malizia A., Bassani L., et al., 2006, MNRAS, 371,821

[333] Corbet R.H.D., Thorstensen J.R., Charles P.A., et al., 1986, MNRAS, 222, 15P

[334] Smith D.M., Heindl W.A., Markwardt C.B., et al., 2006, ApJ, 638, 974

[335] Sakano M., Koyama K., Murakami H., et al., 2002, ApJS, 138,19

[336] Negueruela I., Smith D.M., Harrison T.E., \& Torrejón J.M., 2006, ApJ, 638, 982

[337] Torres M.A.P., Steeghs D., Garcia M.R., et al., 2006, ATel, 784,1

[338] in 't Zand J.J.M., Verbunt F., Kuulkers E., et al., 2002, A\&A, 389, L43

[339] Sidoli L., Belloni T., \& Mereghetti S., 2001, A\&A, 368, 835

[340] Torres M.A.P., Garcia M.R., McClintock J.E., et al., 2004, ATel, 264, 1

[341] Kong A.K.H., 2006, ATel, 745, 1

[342] Kennea J.A., Burrows D.N., Nousek J., \& Gehrels N., 2005, ATel, 617, 1

[343] Rupen M.P., Dhawan V., \& Mioduszewski A.J., 2004, ATel, 335, 1

[344] Martí, J., Mirabel I.F., Chaty S., \& Rodríguez L.F., 2000, A\&A, 363, 184

[345] Gallo E. \& Fender R.P., 2002, MNRAS, 337, 869

[346] Smith D.M., Heindl W.A., \& Swank J.H., 2002, ApJ, 578, L129

[347] White N.E. \& van Paradijs J., 1996, ApJ, 473, L25

[348] Reid M.J. \& Brunthaler A., 2004, ApJ, 616, 872

[349] Bélanger G., Goldwurm A., Renaud M., et al., 2006, ApJ, 636,275

[350] Miller J.M., Raymond J., Homan J., et al., 2006, ApJ, 646, 394

[351] Corbel S., Kaaret P., Fender R.P., et al., 2005, ApJ, 632, 504

[352] Porquet D., Rodriguez J., Corbel S., et al., 2003, A\&A, 406, 299

[353] Revnivtsev M.G., Sunyaev R.A., Varshalovich D.A., et al., 2004, AstL, 30, 382

[354] Wijnands R., Miller J.M., \& Wang Q.D., 2002, ApJ, 579, 422

[355] Kong A.K.H., Wijnands R., \& Homan J., 2005, ATel, 641, 1

[356] Werner N., in 't Zand J.J.M., Natalucci L., et al., 2004, A\&A, 416, 311

[357] Revnivtsev M.G., Churazov E.M., Sazonov S.Y., et al., 2004, A\&A, 425, L49 
[358] Juett A.M., Kaplan D.L., Chakrabarty D., et al., 2005, ATel, 521, 1

[359] Kennea J.A., Burrows D.N., Markwardt C., \& Gehrels N., 2005, ATel, 500, 1

[360] Oosterbroek T., Barret D., Guainazzi M., \& Ford E.C., 2001, A\&A, 366, 138

[361] McClintock J., Murray S., Garcia M., \& Jonker P., 2003, ATel, 205, 1

[362] Bhattacharyya S., Strohmayer T.E., Markwardt C.B., \& Swank J.H., 2006, ApJ, 639, L31

[363] Torii K., Kinugasa K., Katayama K., et al., 1998, ApJ, 508, 854

[364] Paizis A., Nowak M.A., Rodriguez J., et al., 2006, ATel, 907,1

[365] Sidoli L., Parmar A.N., Oosterbroek T., et al., 2001, A\&A, 368,451

[366] in 't Zand J.J.M., Hulleman F., Markwardt C.B., et al., 2003, A\&A, 406, 233

[367] Still M., 2005, ATel, 555, 1

[368] Morris D.C., Burrows D.N., Racusin J., et al., 2005, ATel, 552,1

[369] Türler M., Shaw S.E., Kuulkers E., et al., 2006, ATel, 790, 1

[370] in 't Zand J.J.M., 2005, A\&A, 441, L1

[371] Pellizza L.J., Chaty S., \& Negueruela I., 2006, A\&A, 455, 653

[372] Lutovinov A., Revnivtsev M., Molkov S., \& Sunyaev R., 2005, A\&A, 430, 997

[373] Sidoli L. \& Mereghetti S., 2002, A\&A, 388, 293

[374] Iaria R., di Salvo T., Robba N.R., et al., 2005, A\&A, 439, 575

[375] Hill A.B., Walter R., Knigge C., et al., 2005, A\&A, 439, 255

[376] Combi J.A., Ribó M., Martí J., \& Chaty S., 2006, A\&A, 458,761

[377] Markwardt C.B., Juda M., \& Swank J.H., 2003, IAU Circ., 8095, 2

[378] Falanga M., Bonnet-Bidaud J.M., Poutanen J., et al., 2005, A\&A, 436, 647

[379] Kaplan D.L., Fox D.W., Kulkarni S.R., et al., 2002, ApJ, 564,935

[380] Molkov S., Hurley K., Sunyaev R., et al., 2005, A\&A, 433, L13

[381] Tiengo A., Esposito P., Mereghetti S., et al., 2005, A\&A, 440, L63

[382] Corbel S. \& Eikenberry S.S., 2004, A\&A, 419, 191

[383] Kaspi V.M., Roberts M.E., Vasisht G., et al., 2001, ApJ, 560,371

[384] Roberts M.S.E., Tam C.R., Kaspi V.M., et al., 2003, ApJ, 588,992

[385] Torii K., Tsunemi H., Dotani T., et al., 1999, ApJ, 523, L69

[386] Gotthelf E.V., 2003, ApJ, 591, 361

[387] Ubertini P., Bassani L., Malizia A., et al., 2005, ApJ, 629, L109

[388] Brogan C.L., Gaensler B.M., Gelfand J.D., et al., 2005, ApJ, 629, L105

[389] Ueda Y., Murakami H., Yamaoka K., et al., 2004, ApJ, 609,325

[390] Corbet R.H.D., 2003, ApJ, 595, 1086

[391] Bandyopadhyay R.M., Shahbaz T., Charles P.A., \& Naylor T., 1999, MNRAS, 306, 417

[392] Sguera V., Bird A.J., Dean A.J., et al., 2006, ATel, 873, 1
[393] Farinelli R., Frontera F., Zdziarski A.A., et al., 2005, A\&A, 434, 25

[394] Dhawan M.P.R.V. \& Mioduszewski A.J., 2006, ATel, 721, 1

[395] Miller J.M., Homan J., Steeghs D., \& Wijnands R., 2006, ATel, 746, 1

[396] Sala G. \& Greiner J., 2006, ATel, 791, 1

[397] Gehrels M.S.N., Steeghs D., Torres M.A.P., et al., 2005, ATel, 588, 1

[398] in 't Zand J., Jonker P., Mendez M., \& Markwardt C., 2006, ATel, 915, 1

[399] Kinugasa K., Torii K., Hashimoto Y., et al., 1998, ApJ, 495,435

[400] Voges W., Aschenbach B., Boller T., et al., 2000, IAU Circ., 7432, 1

[401] Migliari S., Fender R.P., Rupen M., et al., 2004, MNRAS, 351,186

[402] Revnivtsev M., Sazonov S., Churazov E., \& Trudolyubov S., 2006, A\&A, 448, L49

[403] Douglas J.N., Bash F.N., Bozyan F.A., et al., 1996, AJ, 111,1945

[404] Juett A.M. \& Chakrabarty D., 2005, ApJ, 627, 926

[405] Iaria R., Di Salvo T., Burderi L., \& Robba N.R., 2001, ApJ, 557, 24

[406] Jonker P.G. \& van der Klis M., 2001, ApJ, 553, L43

[407] Hellier C. \& Mason K.O., 1989, MNRAS, 239, 715

[408] Mason K.O. \& Cordova F.A., 1982, ApJ, 262, 253

[409] Monet D. \& et al. 1998, in The PMM USNO-A2.0 Catalog. (1998), 0-+

[410] Martocchia A., Motch C., \& Negueruela I., 2005, A\&A, 430, 245

[411] Casares J., Ribó M., Ribas I., et al., 2005, MNRAS, 364, 899

[412] Thompson T.W.J., Rothschild R.E., Tomsick J.A., \& Marshall H.L., 2005, ApJ, 634, 1261

[413] Homer L., Charles P.A., \& O'Donoghue D., 1998, MNRAS, 298, 497

[414] Kong A.K.H., Homer L., Kuulkers E., et al., 2000, MNRAS, 311, 405

[415] Safi-Harb S., Harrus I.M., Petre R., et al., 2001, ApJ, 561, 308

[416] Camilo F., Ransom S.M., Gaensler B.M., et al., 2006, ApJ, 637,456

[417] Schmitz M., 1991, NED Team Report, 1, 1 (1991), 1, 1

[418] de Rosa A., Piro L., Tramacere A., et al., 2005, A\&A, 438, 121

[419] Grandi P., Urry C.M., \& Maraschi L., 2002, New A Rev., 46, 221

[420] Parmar A.N., Oosterbroek T., Sidoli L., Stella L., \& Frontera F., 2001, A\&A, 380, 490

[421] Deutsch E.W., Margon B., \& Anderson S.F., 2000, ApJ, 530, L21

[422] Malizia A., Bassani L., Stephen J.B., et al., 2005, ApJ, 630, L157

[423] Foster R.S., Cadwell B.J., Wolszczan A., \& Anderson S.B., 1995, ApJ, 454, 826

[424] Molkov S.V., Cherepashchuk A.M., Lutovinov A.A., et al., 2004, AstL, 30, 534

[425] Halpern J.P., Gotthelf E.V., Helfand D.J., et al., 2004, ATel, 289,1

[426] Bamba A., Yokogawa J., Ueno M., et al., 2001, PASJ, 53, 1179

[427] Wachter S., Patel S.K., Kouveliotou C., et al., 2004, ApJ, 615,887 
[428] Morii M., Sato R., Kataoka J., \& Kawai N., 2003, PASJ, 55 , L45

[429] Vasisht G. \& Gotthelf E.V., 1997, ApJ, 486, L129

[430] Sanbonmatsu K.Y. \& Helfand D.J., 1992, AJ, 104, 2189

[431] Sguera V., Bird A.J., Dean A.J., et al., 2007, A\&A, 462, 695

[432] Coe M.J., Fabregat J., Negueruela I., et al., 1996, MNRAS, 281, 333

[433] Piraino S., Santangelo A., Segreto A., et al., 2000, A\&A, 357,501

[434] Israel G.L., Negueruela I., Campana S., et al., 2001, A\&A, 371,1018

[435] Gotthelf E.V., Vasisht G., Boylan-Kolchin M., \& Torii K., 2000, ApJ, 542, L37

[436] Helfand D.J., Collins B.F., \& Gotthelf E.V., 2003, ApJ, 582,783

[437] Livingstone M.A., Kaspi V.M., Gotthelf E.V., \& Kuiper L., 2006, ApJ, 647, 1286

[438] Becker R.H. \& Helfand D.J., 1984, ApJ, 283, 154

[439] Soffitta P., Tomsick J.A., Harmon B.A., et al., 1998, ApJ, 494, L203

[440] Lutovinov A.A. \& Revnivtsev M.G., 2003, AstL, 29, 719

[441] Beuermann K., Harrison T.E., McArthur B.E., et al., 2004, A\&A, 419, 291

[442] Corbet R.H.D. \& Mukai K., 2002, ApJ, 577, 923

[443] Corbet R.H.D., Marshall F.E., Peele A.G., \& Takeshima T., 1999, ApJ, 517, 956

[444] Harris D.E., Forman W., Gioa I.M., et al., 1996, VizieR Online Data Catalog, 9013, 0

[445] Bikmaev I.F., Sunyaev R.A., Revnivtsev M.G., \& Burenin R.A., 2005, AstL, in press, astro-ph/0511405

[446] Paul B. \& Rao A.R., 1998, A\&A, 337, 815

[447] Takeshima T., Corbet R.H.D., Marshall F.E., et al., 1998, IAU Circ., 6826, 1

[448] Rupen M.P., Mioduszewski A.J., \& Dhawan V., 2005, ATel, 530, 1

[449] Campana S., 2005, ATel, 535, 1

[450] Kaaret P., Morgan E.H., Vanderspek R., \& Tomsick J.A., 2006, ApJ, 638, 963

[451] Kawai N. \& Suzuki M., 2005, ATel, 534, 1

[452] Galloway D.K., Remillard R., \& Morgan E., 2003, IAU Circ., 8081, 2

[453] Galloway D.K., Wang Z., \& Morgan E.H., 2005, ApJ, 635, 1217

[454] Frail D.A., Kulkarni S.R., \& Bloom J.S., 1999, Nature, 398, 127

[455] Esposito P., Mereghetti S., Tiengo A., et al., 2007, A\&A, 461,605

[456] Vrba F.J., Henden A.A., Luginbuhl C.B., et al., 2000, ApJ, 533, L17

[457] Rupen M.P., Dhawan V., \& Mioduszewski A.J., 2002, IAU Circ., 7874, 1

[458] in 't Zand J.J.M., Miller J.M., Oosterbroek T., \& Parmar A.N., 2002, A\&A, 394, 553

[459] Chaty S., Mignani R.P., \& Israel G.L., 2006, MNRAS, 365,1387

[460] Cusumano G., di Salvo T., Burderi L., et al., 1998, A\&A, 338, L79

[461] Baykal A., İnam S.C., \& Beklen E., 2006, MNRAS, 369, 1760

[462] in 't Zand J.J.M., Baykal A., \& Strohmayer T.E., 1998, ApJ, 496, 386

[463] Cox N.L.J., Kaper L., \& Mokiem M.R., 2005, A\&A, 436, 661
[464] Levine A.M., Rappaport S., Remillard R., \& Savcheva A., 2004, ApJ, 617, 1284

[465] Morel T. \& Grosdidier Y., 2005, MNRAS, 356, 665

[466] Wijnands R., Maitra D., Bailyn C., \& Linares M., 2006, ATel, 871, 1

[467] Chevalier C. \& Ilovaisky S.A., 1991, A\&A, 251, L11

[468] Brinkmann W., Kotani T., \& Kawai N., 2005, A\&A, 431, 575

[469] Blundell K.M. \& Bowler M.G., 2004, ApJ, 616, L159

[470] in 't Zand J.J.M., Jonker P.G., Nelemans G., et al., 2006, A\&A, 448, 1101

[471] Corbet R.H.D., Hannikainen D.C., \& Remillard R., 2004, ATel, 269, 1

[472] Martocchia A., Matt G., Belloni T., et al., 2006, A\&A, 448,677

[473] Harlaftis E.T. \& Greiner J., 2004, A\&A, 414, L13

[474] Juett A.M. \& Chakrabarty D., 2006, ApJ, 646, 493

[475] Falanga M., Belloni T., \& Campana S., 2006, A\&A, 456, L5

[476] Sambruna R.M., 1997, ApJ, 487, 536

[477] Véron-Cetty M.-P. \& Véron P., 2003, A\&A, 412, 399

[478] Wang J., Wei J.Y., \& He X.T., 2006, ApJ, 638, 106

[479] Andronov I.L., Baklanov A.V., \& Burwitz V., 2006, A\&A, 452,941

[480] Rana V.R., Singh K.P., Barrett P.E., \& Buckley D.A.H., 2005, ApJ, 625, 351

[481] Naik S., Callanan P.J., Paul B., \& Dotani T., 2006, ApJ, 647,1293

[482] Galloway D.K., Morgan E.H., \& Levine A.M., 2004, ApJ, 613,1164

[483] Tsygankov S.S. \& Lutovinov A.A., 2005, AstL, 31, 88

[484] Evans D.A., Worrall D.M., Hardcastle M.J., et al., 2006, ApJ, 642, 96

[485] Liu F.K. \& Zhang Y.H., 2002, A\&A, 381, 757

[486] Masetti N., Orlandini M., Palazzi E., et al., 2006, A\&A, 453, 295

[487] Mattana F., Götz D., Falanga M., et al., 2006, A\&A, 460, L1

[488] Schulz N.S., Cui W., Canizares C.R., et al., 2002, ApJ, 565,1141

[489] Ziólkowski J., 2005, MNRAS, 358, 851

[490] Zhou H.-Y. \& Wang T.-G., 2002, Chinese J. Astron. Astrophys., 2, 501

[491] NED Team, 1992, NED Team Report, 1, 1 (1992), 1, 1

[492] Bykov A.M., Krassilchtchikov A.M., Uvarov Y.A., et al., 2006, ApJ, 649, L21

[493] Reig P. \& Coe M.J., 1999, MNRAS, 302, 700

[494] Camero Arranz A., Wilson C.A., Connell P., et al., 2005, A\&A, 441, 261

[495] Wilson C.A., Finger M.H., Coe M.J., et al., 2002, ApJ, 570,287

[496] Weekes T.C. \& Geary J.C., 1982, PASP, 94, 708

[497] Stark M.J. \& Saia M., 2003, ApJ, 587, L101

[498] Lara L., Cotton W.D., Feretti L., et al., 2001, A\&A, 370, 409

[499] Ballantyne D.R., 2005, MNRAS, 362, 1183

[500] Cutri R.M., Skrutskie M.F., van Dyk S., et al., 2003, The IRSA 2MASS All-Sky Point Source Catalog, NASA/IPAC Infrared Science Archive. (http://irsa.ipac.caltech.edu/applications/Gator/)

[501] Baykal A., Stark M.J., \& Swank J.H., 2002, ApJ, 569, 903 [502] Sidoli L., Mereghetti S., Larsson S., et al., 2005, A\&A, 440,1033 
[503] Falcone A., Tueller J., Markwardt C., et al., 2006, ATel, 846,1

[504] Thorstensen J.R. \& Taylor C.J., 2001, MNRAS, 326, 1235

[505] Halpern J.P., 2006, ATel, 847, 1

[506] Bonnet-Bidaud J.M., Mouchet M., de Martino D., et al., 2006, A\&A, 445, 1037

[507] Motch C., Guillout P., Haberl F., et al., 1997, A\&AS, 122, 201

[508] Mauche C.W., 2004, ApJ, 610, 422

[509] Harrison T.E., McNamara B.J., Szkody P., \& Gilliland R.L., 2000, AJ, 120, 2649

[510] Costantini E., Freyberg M.J., \& Predehl P., 2005, A\&A, 444, 187

[511] Casares J., Charles P.A., \& Kuulkers E., 1998, ApJ, 493, L39

[512] Stickel M., Lemke D., Klaas U., et al., 2004, A\&A, 422, 39

[513] Dadina M., Bassani L., Cappi M., et al., 2001, A\&A, 370, 70

[514] Ravasio M., Tagliaferri G., Ghisellini G., et al., 2003, A\&A, 408, 479

[515] Hog E., Kuzmin A., Bastian U., et al., 1998, A\&A, 335, L65

[516] Masetti N., Dal Fiume D., Amati L., et al., 2004, A\&A, 423,311

[517] Ribó M., Negueruela I., Blay P., et al., 2006, A\&A, 449, 687

[518] Blay P., Negueruela I., Reig P., et al., 2006, A\&A, 446, 1095

[519] Patterson J., Kemp J., Richman H.R., et al., 1998, PASP, 110,415

[520] Barrett P., 1996, PASP, 108, 412

[521] Gibson R.R., Marshall H.L., Canizares C.R., \& Lee J.C., 2005, ApJ, 627, 83

[522] Scott J.E., Kriss G.A., Lee J.C., et al., 2005, ApJ, 634, 193

[523] George I.M., Turner T.J., Netzer H., et al., 1998, ApJS, 114,73

[524] Hwang U., Laming J.M., Badenes C., et al., 2004, ApJ, 615, L117

[525] Reed J.E., Hester J.J., Fabian A.C., \& Winkler P.F., 1995, ApJ, 440, 706 